\title{
Quantum knots and mosaics
}

\author{
Samuel J. Lomonaco • Louis H. Kauffman
}

(C) Springer Science+Business Media, LLC 2008

\begin{abstract}
In this paper, we give a precise and workable definition of a quantum knot system, the states of which are called quantum knots. This definition can be viewed as a blueprint for the construction of an actual physical quantum system. Moreover, this definition of a quantum knot system is intended to represent the "quantum embodiment" of a closed knotted physical piece of rope. A quantum knot, as a state of this system, represents the state of such a knotted closed piece of rope, i.e., the particular spatial configuration of the knot tied in the rope. Associated with a quantum knot system is a group of unitary transformations, called the ambient group, which represents all possible ways of moving the rope around (without cutting the rope, and without letting the rope pass through itself.) Of course, unlike a classical closed piece of rope, a quantum knot can exhibit non-classical behavior, such as quantum superposition and quantum entanglement. This raises some interesting and puzzling questions about the relation between topological and quantum entanglement. The knot type of a quantum knot is simply the orbit of the quantum knot under the action of the ambient group. We investigate quantum observables which are invariants of quantum knot type. We also study the Hamiltonians associated with the generators of the ambient group, and briefly look at the quantum tunneling of overcrossings into undercrossings. A basic building block in this paper is a mosaic system which is a formal (rewriting) system of symbol strings. We conjecture that this formal system fully captures in an axiomatic way all of the properties of tame knot theory.
\end{abstract}

\footnotetext{
S. J. Lomonaco (凶)

University of Maryland Baltimore County (UMBC), Baltimore, MD 21250, USA

e-mail: lomonaco@umbc.edu

URL: http://www.csee.umbc.edu/ lomonaco

L. H. Kauffman

University of Illinois at Chicago (UIC), Chicago, IL 60607-7045, USA

e-mail: kauffman@uic.edu

URL: http://www.math.uic.edu/ kauffman
} 
Keywords Quantum knots - Knots - Knot theory · Quantum computation ·

Quantum algorithms · Quantum vortices

Mathematics Subject Classification (2000) Primary 81P68 - 57M25 - 81P15 .

$57 \mathrm{M} 27 \cdot$ Secondary 20C35

\section{Introduction}

The objective of this paper is to set the foundation for a research program on quantum knots. ${ }^{1}$

For simplicity of exposition, we will throughout this paper frequently use the term "knot" to mean either a knot or a link. ${ }^{2}$

In part 1 of this paper, we create a formal system $(\mathbb{K}, \mathbb{A})$ consisting of

(1) A graded set $\mathbb{K}$ of symbol strings, called knot mosaics, and

(2) A graded subgroup $\mathbb{A}$, called the knot mosaic ambient group, of the group of all permutations of the set of knot mosaics $\mathbb{K}$.

We conjecture that the formal system $(\mathbb{K}, \mathbb{A})$ fully captures the entire structure of tame knot theory.

Three examples of knot mosaics are given below:
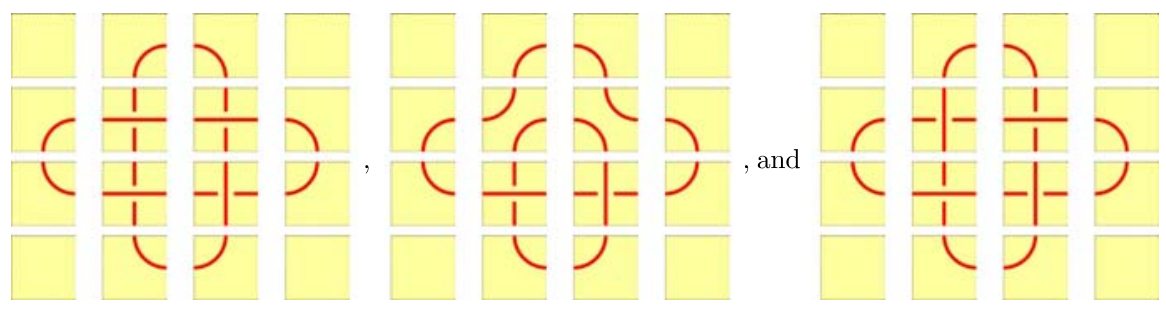

Each of these knot mosaics is a string made up of the following 11 symbols
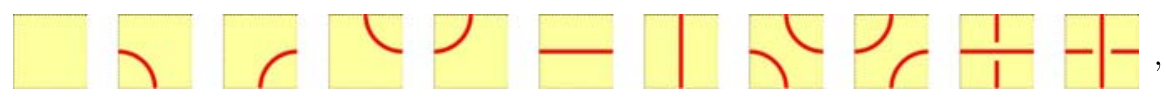

called mosaic tiles.

An example of an element in the mosaic ambient group $\mathbb{A}$ is the mosaic Reidemeister 1 move illustrated below:

$$
N \stackrel{(0,1)}{\longleftrightarrow} N^{\prime}=\frac{\Gamma}{\frac{1}{1}} \frac{1}{\frac{1}{1}} \stackrel{(0,1)}{\longleftarrow}
$$

\footnotetext{
${ }_{1}^{1}$ A PowerPoint presentation of this paper can be found at http://www.csee.umbc.edu/ lomonaco/Lectures. html.

${ }^{2}$ For references on knot theory, see for example $[4,10,13,20]$.
} 
This mosaic Reidemeister 1 move $N \stackrel{(0,1)}{\longleftrightarrow} N^{\prime}$ is a permutation which is the product of disjoint transpositions, as illustrated by observing that the Reidemeister 1 move $N \stackrel{(0,1)}{\longleftrightarrow} N^{\prime}$ interchanges the following two knot mosaics:

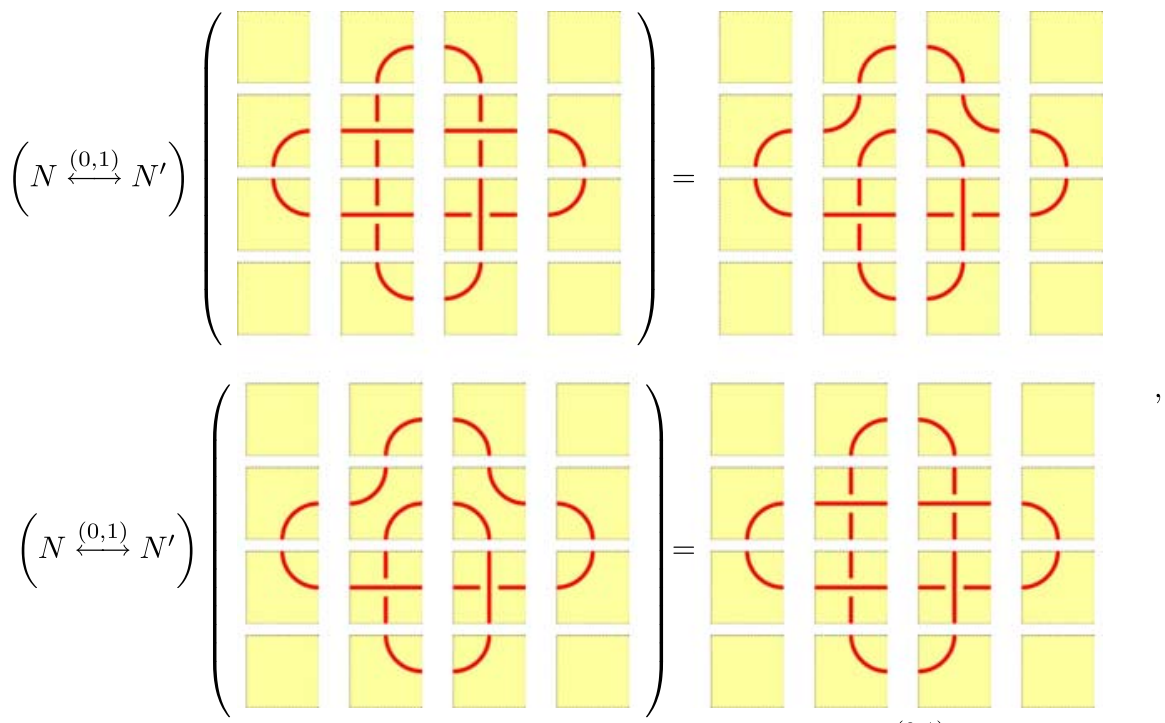

Knot mosaics are interchanged by $N \stackrel{(0,1)}{\longleftrightarrow} N^{\prime}$

while it leaves the following mosaic unchanged:

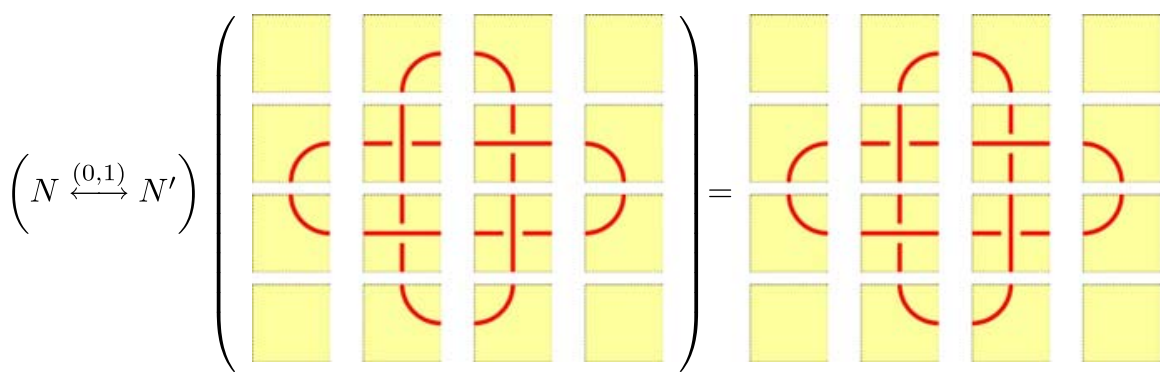

The knot mosaic is left fixed by $N \stackrel{(0,1)}{\longleftrightarrow} N^{\prime}$

In part 2, the formal system $(\mathbb{K}, \mathbb{A})$ is used to define a quantum knot system $Q(\mathcal{K}, \mathbb{A})$, which is a nested sequence of quantum systems consisting of

(1) A graded Hilbert space $\mathcal{K}$, called the quantum knot state space, defined by an orthonormal basis labelled by and in one-to-one correspondence with the set of knot mosaics $\mathbb{K}$, and

(2) An associated graded control group, also called the ambient group, and also denoted by $\mathbb{A}$. The ambient group $\mathbb{A}$ is a discrete subgroup of the group $U(\mathcal{K})$ of all unitary transformations on the Hilbert space $\mathcal{K}$. 
A quantum knot is simply a state of the quantum knot system, i.e., an element of the quantum knot state space $\mathcal{K}$. Quantum knot type is defined as the orbit of the quantum knot under the action of the ambient group $\mathbb{A}$.

Once having defined what is meant by a quantum knot, we then proceed to find the Hamiltonians associated with the generators of the ambient group $\mathbb{A}$, and to study the quantum dynamics induced by Schroedinger's equation. We move on to discuss other Hamiltonians, such as for example those associated with overcrossings quantum tunnelling into undercrossings. We also study a class of quantum observables which are quantum knot invariants.

We should mention that, if one selects a fixed upper bound $n$ on knot complexity (i.e., a fixed upper bound on the edge length $n$ of the knot $n$-mosaics under consideration), then a quantum knot system $Q\left(\mathcal{K}^{(n)}, \mathbb{A}(n)\right)$ is a blueprint for the construction of an actual physical quantum system. Quantum knots could possibly be used to simulate and to predict the behavior of quantum vortices that appear both in liquid helium II and in the Bose-Einstein condensate. They might also possibly be a mathematical model for gaining some insight into the charge quantification that is manifest in the fractional quantum Hall effect.

In the conclusion, we list a number of open questions and possible future research directions. A complete table of all knot 3-mosaics is given in Appendix A. Finally, in Appendix B, we briefly outline the theory of and the construction of oriented knot mosaics and oriented quantum knots.

The motivating intuition for the above mathematical construct $Q(\mathcal{K}, \mathbb{A})$ is as follows: A quantum knot system is intended to represent the "quantum embodiment" of a closed knotted physical piece of rope. A quantum knot is meant to represent the state of the knotted rope, i.e., the particular spatial configuration of the knot tied in the rope. The elements of the ambient unitary group are intended to represent all possible ways of moving the rope around (without cutting the rope, and without letting it pass through itself). The quantum system is necessarily a nested set of quantum systems because one must use longer and longer pieces of rope to tie knots of greater and greater complexity.

Of course, unlike classical knotted pieces of rope, quantum knots can also represent the quantum superpositions (and also the quantum entanglements) of a number of knotted pieces of rope. This raises an interesting question about the relation between topological entanglement and quantum entanglement.

\section{Part 1: knot mosaics}

\subsection{Unoriented knot mosaics}

Let $\mathbb{T}^{(u)}$ denote the set of the following 11 symbols
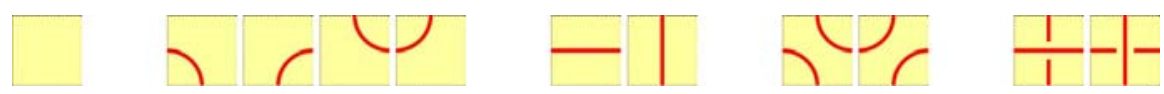

called (unoriented) tiles. We often will also denote these tiles respectively by the following symbols 


$$
\begin{array}{lllllllllll}
T_{0}^{(u)} & T_{1}^{(u)} & T_{2}^{(u)} & T_{3}^{(u)} & T_{4}^{(u)} & T_{5}^{(u)} & T_{6}^{(u)} & T_{7}^{(u)} & T_{8}^{(u)} & T_{9}^{(u)} & T_{10}^{(u)} .
\end{array}
$$

Moreover, we will frequently omit the superscript ' $(u)$ ' (standing for 'unoriented') when it can be understood from context.

Remark 1 Please note that up to rotation there are exactly 5 distinct unoriented tiles. The above unoriented tiles are grouped according to rotational equivalence.

Definition 1 Let $n$ be a positive integer. We define an (unoriented) $n$-mosaic as an $n \times n$ matrix $M=\left(M_{i j}\right)=\left(T_{k(i, j)}\right)$ of (unoriented) tiles with rows and columns indexed from 0 to $n-1$. We denote the set of $n$-mosaics by $\mathbb{M}^{(n)}$.

Two examples of unoriented 4-mosaics are shown below:
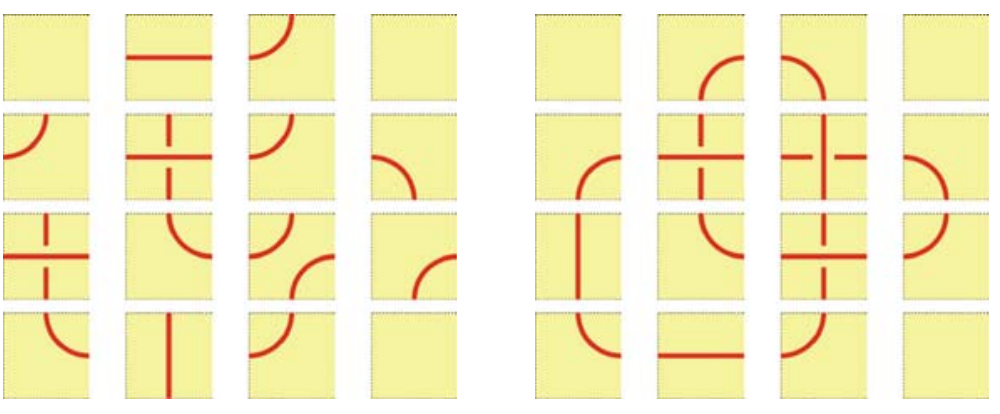

We now proceed to define what is meant by a knot mosaic:

A connection point of a tile is defined as the midpoint of a tile edge which is also the endpoint of a curve drawn on the tile.

Examples of tile connection points are illustrated in Fig. 1 below:

We say that two tiles in a mosaic are contiguous if they lie immediately next to each other in either the same row or the same column. An unoriented tile within a mosaic is said to be suitably connected if each of its connection points touches a connection point of a contiguous tile.
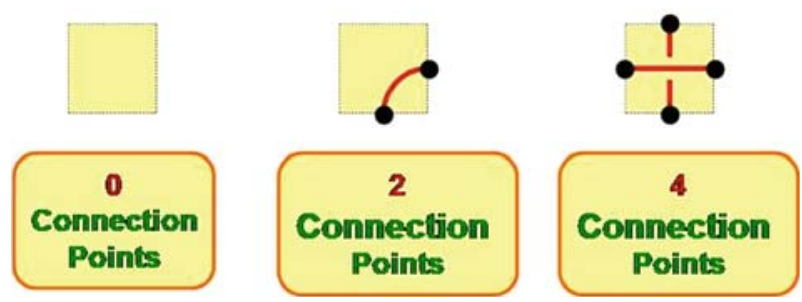

Fig. 1 Tile connection points 
Definition 2 An (unoriented) knot n-mosaic is a mosaic in which all tiles are suitably connected. We let $\mathbb{K}^{(n)}$ denote the subset of $\mathbb{M}^{(n)}$ of all knot $n$-mosaics. ${ }^{3}$

The previous two 4-mosaics shown above are examples respectively of a non-knot 4-mosaic and a knot 4-mosaic. Other examples of knot (or link) mosaics are the Hopf link 4-mosaic, the figure eight knot 5-mosaic, and the Borromean rings 6-mosaic, respectively illustrated below:
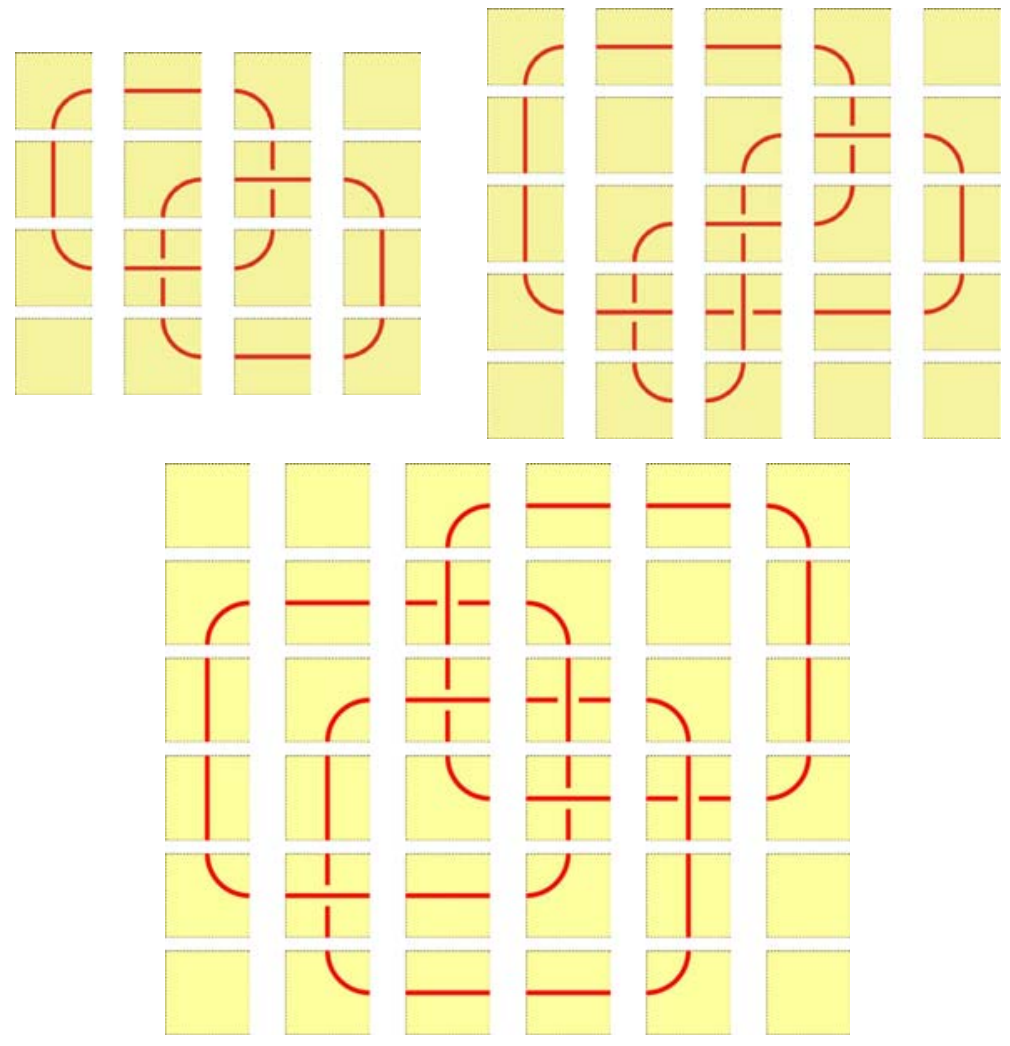

\subsection{Mosaic moves}

We now continue with our program of using mosaics to create a formal model of (tame) knot theory.

Definition 3 Let $k$ and $n$ be positive integers such that $k \leq n$. A $k$-mosaic $N$ is said to be a $k$-submosaic of an $n$-mosaic $M$ if it is a $k \times k$ submatrix of $M$. The $k$-submosaic $N$ is said to be at location $(i, j)$ in the $n$-mosaic $M$ if the top left entry of $N$ lies in row $i$ and column $j$ of $M$. Obviously, the set of possible locations for a $k$-submosaic

\footnotetext{
3 We remind the reader of the following statement made at the beginning of the introduction of this paper: For simplicity of exposition, we will throughout this paper frequently use the term "knot" to mean either a knot or a link.
} 
of an $n$-mosaic is $\{(i, j): 0 \leq i, j \leq n-k\}$. Moreover, there are exactly $(n-k+1)^{2}$ different locations. Let $M^{(k: i, j)}$ denote the $k$-submosaic of $M$ at location $(i, j)$.

For example, the 3-mosaic

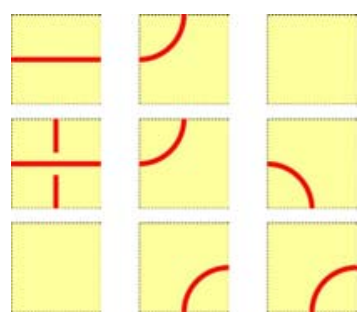

is the submosaic $M^{(3: 0,1)}$ of the 4-mosaic

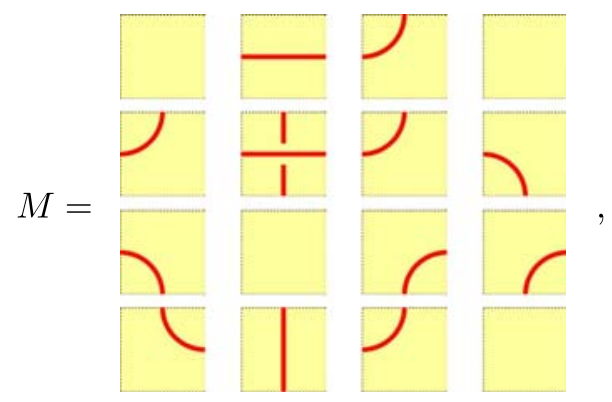

and the 2-mosaic

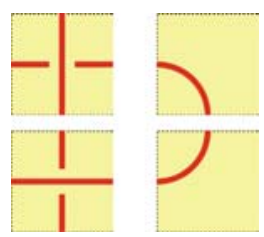

is the submosaic $M^{(2: 1,2)}$ of the 4-mosaic

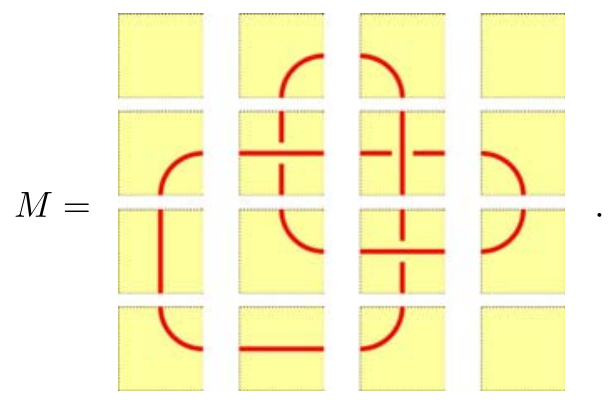

Definition 4 Let $k$ and $n$ be positive integers such that $k \leq n$. For any two $k$-mosaics $N$ and $N^{\prime}$, we define a $k$-move at location $(i, j)$ on the set of $n$-mosaics $\mathbb{M}^{(n)}$, denoted by 


$$
N \stackrel{(i, j)}{\longleftrightarrow} N^{\prime}
$$

as the map from $\mathbb{M}^{(n)}$ to $\mathbb{M}^{(n)}$ defined by

$$
\left(N \stackrel{(i, j)}{\longleftrightarrow} N^{\prime}\right)(M)= \begin{cases}M \text { with } M^{(k: i, j)} & \text { replaced by } N^{\prime} \text { if } M^{(k: i, j)}=N \\ M \text { with } M^{(k: i, j)} & \text { replaced by } N \text { if } M^{(k: i, j)}=N^{\prime} \\ M & \text { otherwise }\end{cases}
$$

As an example, consider the 2-move $N \stackrel{(0,1)}{\longleftrightarrow} N^{\prime}$ defined by

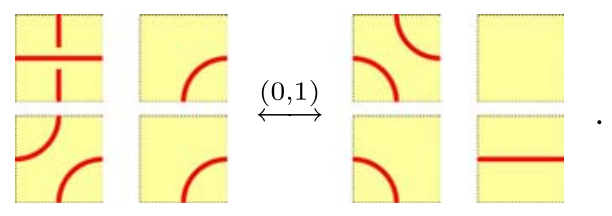

Then,

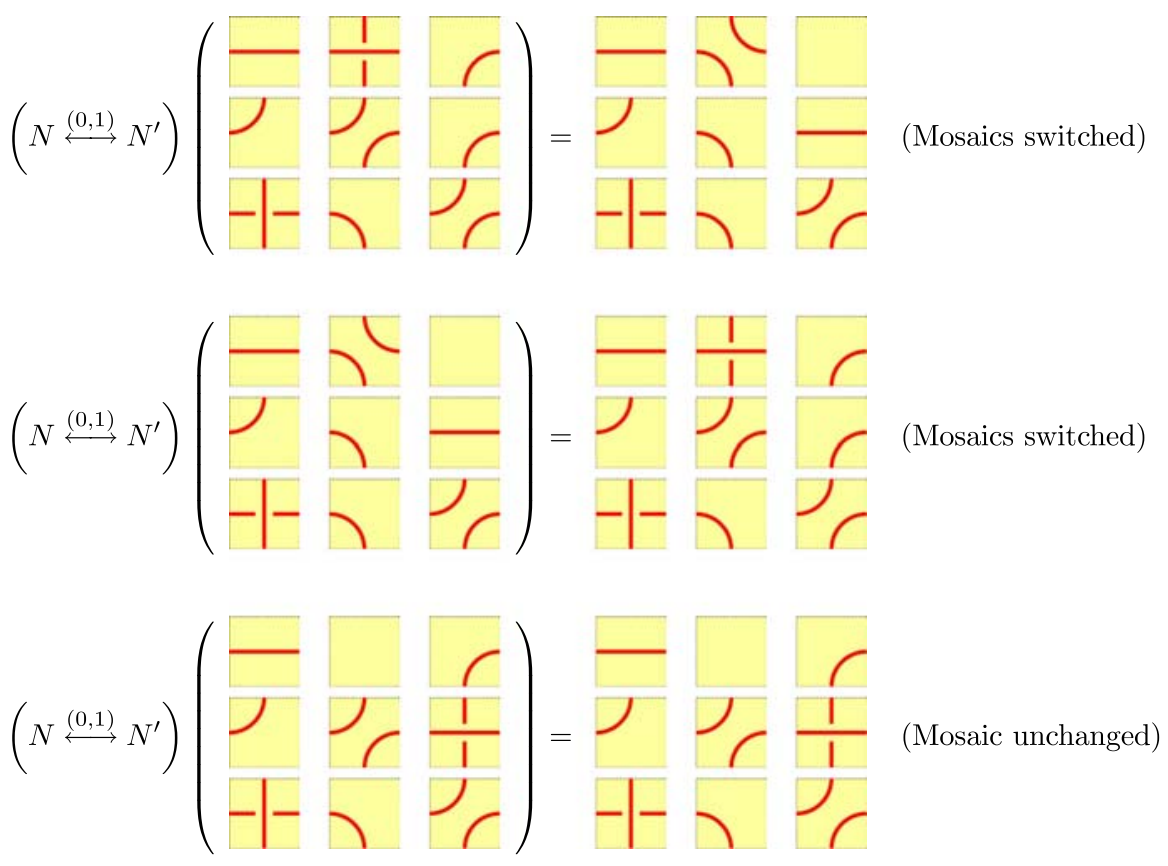

The following proposition is an almost immediate consequence of the definition of a $k$-move:

Proposition 1 Each $k$-move $N \stackrel{(i, j)}{\longleftrightarrow} N^{\prime}$ is a permutation of $\mathbb{M}^{(n)}$. In fact, it is a permutation which is the product of disjoint transpositions. 


\subsection{Three important notational conventions}

For the purpose of achieving clarity of exposition and of simplifying the exposition as much as possible, we adopt the following three nondeterministic notational conventions which will eliminate a great deal of combinatorial clutter:

Notational Convention 1 We will use each of the following tiles
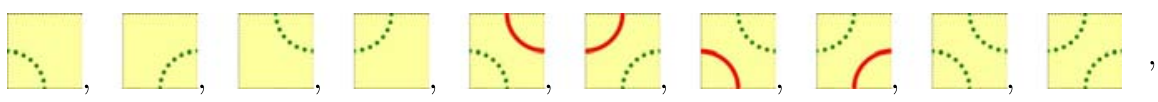

called nondeterministic tiles, to denote either both or any one (depending on context) of two possible tiles.

For example, the nondeterministic tile two tiles and

Notational Convention 2 It is to be understood that each mosaic move $N \stackrel{(i, j)}{\longleftrightarrow} N^{\prime}$ denotes either all or any one (depending on context) of the moves obtained by simultaneously rotating $N$ and $N^{\prime}$ about their respective centers by $0,90,180$, or 270 degrees.

For example,
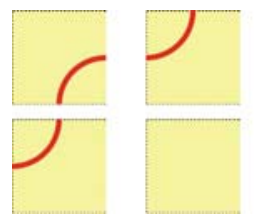

$\stackrel{(0,1)}{\longleftrightarrow}$
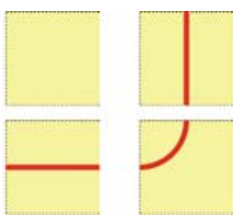

represents either all or any one (depending on context) of the following four 2-moves:
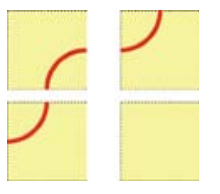

$\stackrel{(0,1)}{\longleftrightarrow}$
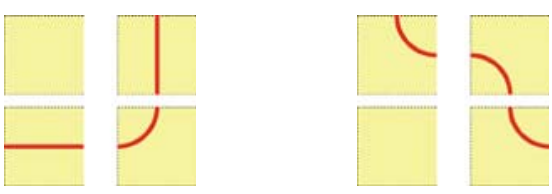

$\stackrel{(0,1)}{\longleftrightarrow}$
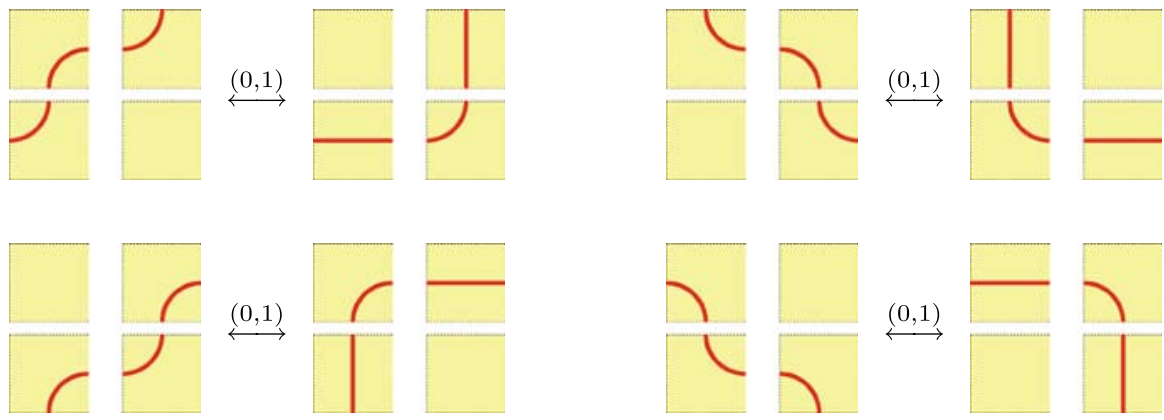

$\stackrel{(0,1)}{\longleftrightarrow}$
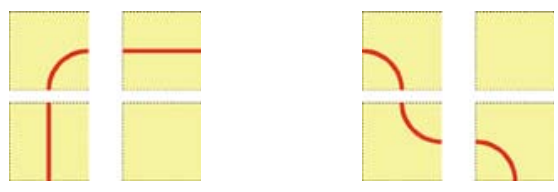

\section{$\stackrel{(0,1)}{\longleftrightarrow}$}
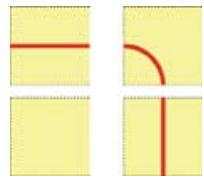

As our final notational convention, we have:

Notational Convention 3 Finally, we omit the location superscript $(i, j)$, and write $N \longleftrightarrow N^{\prime}$ to denote either all or any one (depending on context) of the possible locations.

Caveat: We caution the reader that throughout the remainder of this paper, we will be using all of the above nondeterministic notational conventions. 
2.4 The planar isotopy moves on knot mosaics

As an analog to the planar isotopy moves for standard knot diagrams, we define for mosaics the 11 mosaic planar isotopy moves given below:
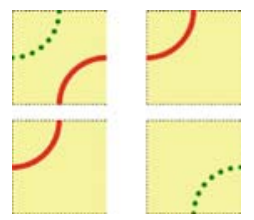

$\longleftrightarrow$

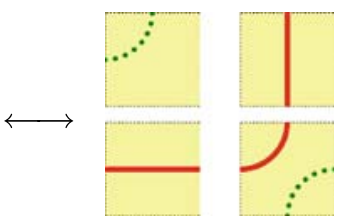

$P_{1}$
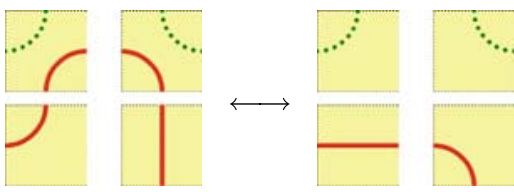

$P_{3}$
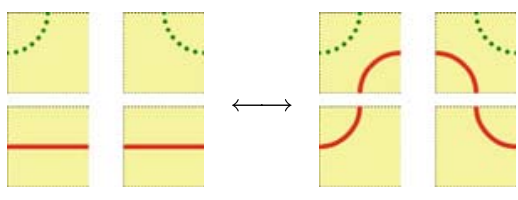

$P_{4}$
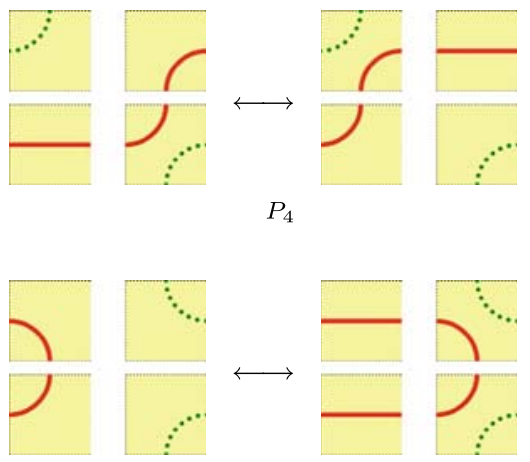

$P_{6}$
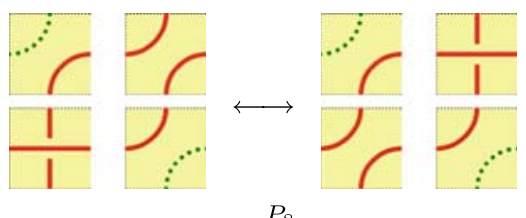

$P_{8}$
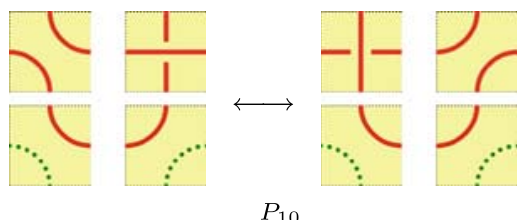

$P_{10}$

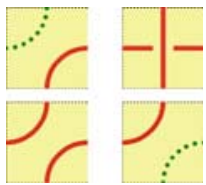

$P_{9}$

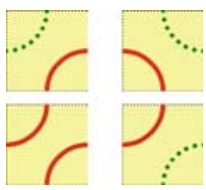

$P_{7}$
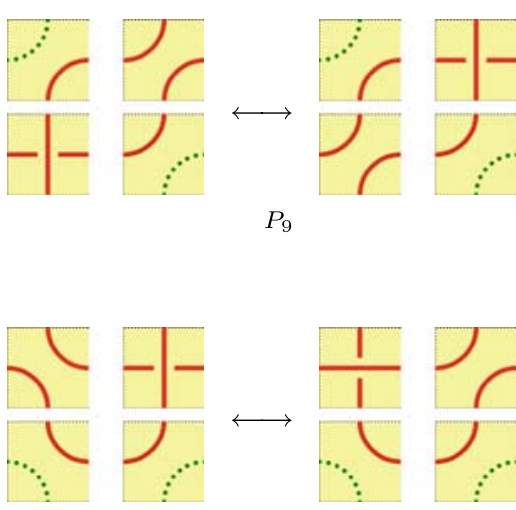
The above set of 11 planar isotopy moves was found by an exhaustive enumeration of all 2-mosaic moves corresponding to topological planar isotopy moves. The completeness of this set of moves, i.e., that every planar isotopy moves for mosaics is a composition of a finite sequence of the above planar isotopy moves, is addressed in Sect. 2.7 of this paper.

\subsection{The Reidemeister moves on knot mosaics}

As an analog to the Reidemeister moves for standard knot diagrams, we create for mosaics the mosaic Reidemeister moves.

The mosaic Reidemeister 1 moves are the following:
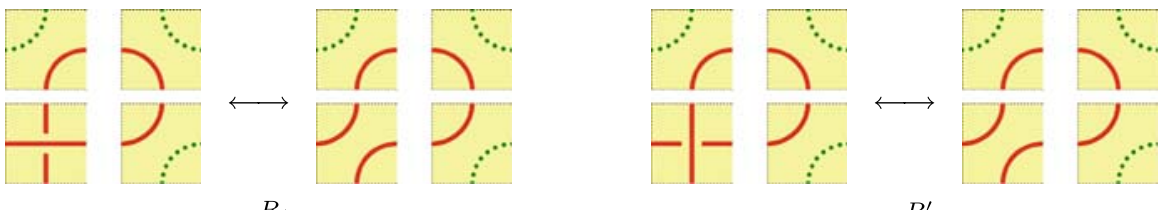

And the mosaic Reidemeister 2 moves are given below:
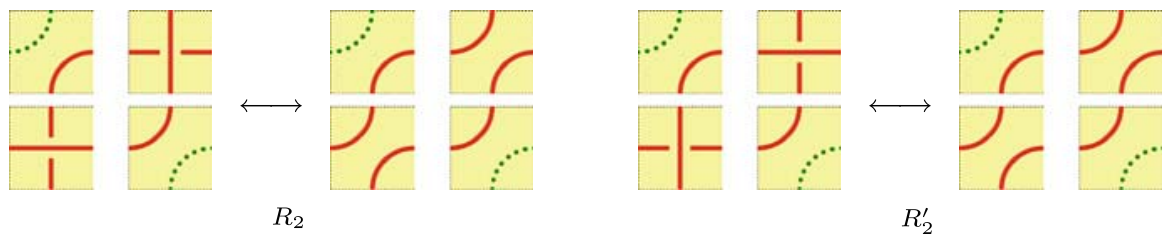

$R_{2}$
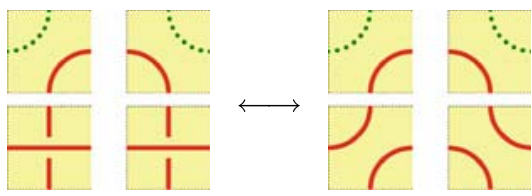

$R_{2}^{\prime \prime}$
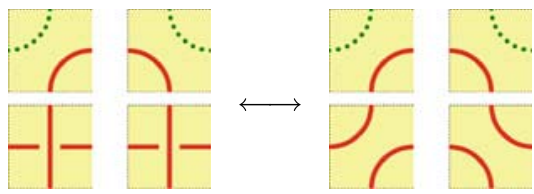

$R_{2}^{\prime \prime \prime}$

For describing the mosaic Reidemeister 3 moves, we will use for simplicity of exposition the following two additional notational conventions:

Notational Convention 4 We will make use of each of the following tiles
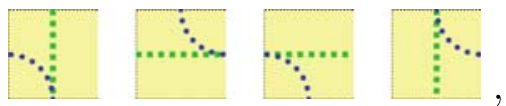

also called nondeterministic tiles, to denote either one of two possible tiles. ${ }^{4}$

For example, the nondeterministic tile $\cdots$ denotes either of the following two tiles

\footnotetext{
${ }^{4}$ Please note that each of these newly introduced non-deterministic tiles denotes one of two possible deterministic tiles. On the other hand, the non-deterministic tiles introduced in Sect. 2.3 denote one or all of two possible deterministic tiles, depending on context.
} 


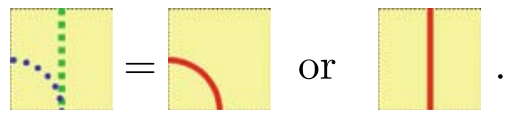

Notational Convention 5 Nondeterministic tiles labeled by the same letter are synchronized as follows:

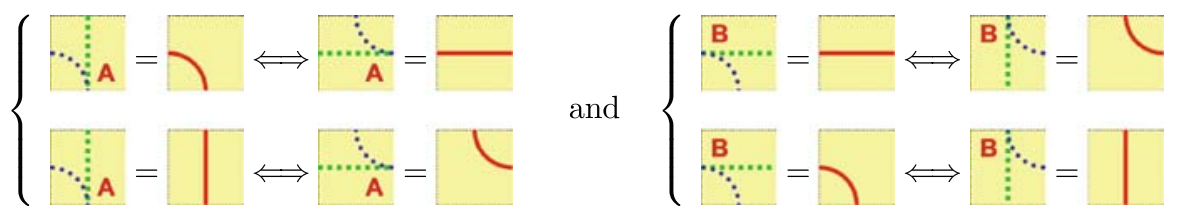

With these two additional notational conventions, the mosaic Reidemeister 3 moves are given below:

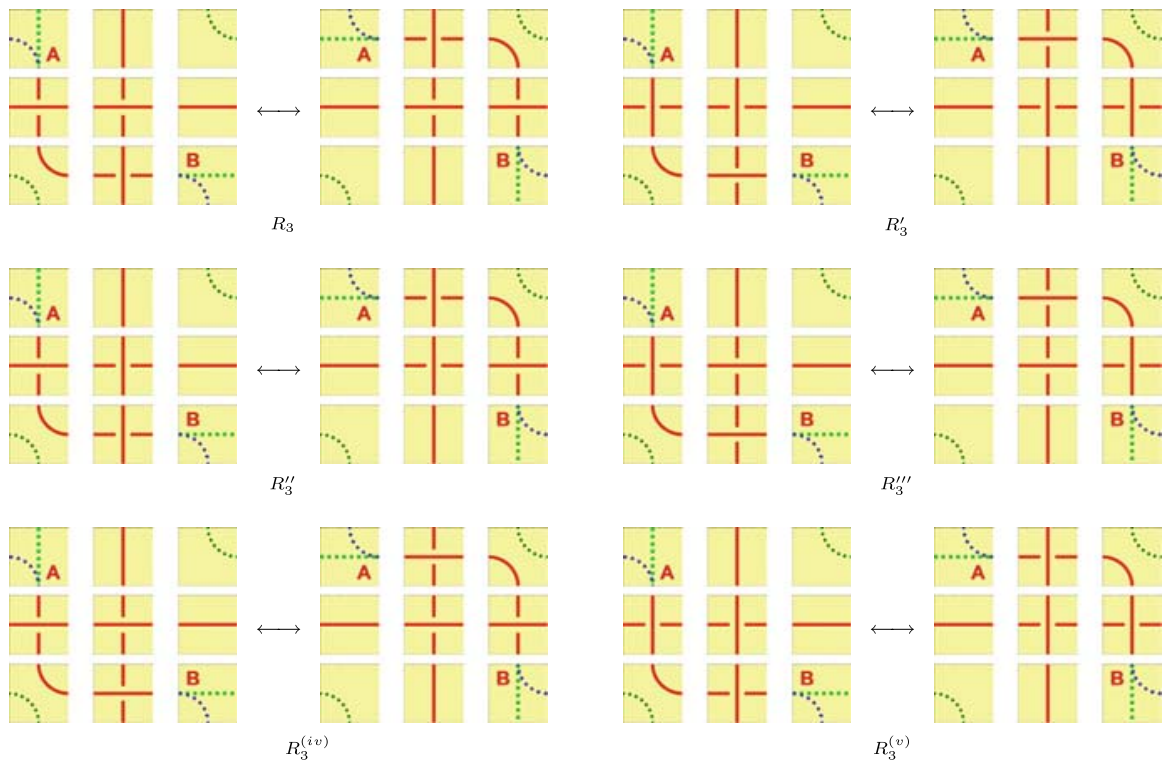

As noted in a previous section, all mosaic moves are permutations on the set of mosaics $\mathbb{M}^{(n)}$. In particular, the planar isotopy moves and the Reidemeister moves lie in the permutation group of the set of mosaics. It easily follows that the planar isotopy moves and the Reidemeister moves also lie in the group of all permutations of the set of knot mosaics $\mathbb{K}^{(n)}$. Hence, we can make the following definition:

Definition 5 We define the (knot mosaic) ambient group $\mathbb{A}(n)$ as the group of all permutations of the set of knot $n$-mosaics $\mathbb{K}^{(n)}$ generated by the mosaic planar isotopy and the mosaic Reidemeister moves.

Remark 2 It follows from a previous proposition that the mosaic planar isotopy moves and Reidemeister moves, as permutations, are each the product of disjoint transpositions.

The completeness of the set of planar isotopy and Reidemeister moves is addressed in Sect. 2.7 of this paper. 


\subsection{Knot mosaic type}

We now are prepared to define the analog of knot type for mosaics.

We define the mosaic injection

$$
\begin{aligned}
\iota: \mathbb{M}^{(n)} & \longrightarrow \mathbb{M}^{(n+1)} \\
M^{(n)} & \longmapsto M^{(n+1)}
\end{aligned}
$$

as

$$
M_{i j}^{(n+1)}= \begin{cases}M_{i j}^{(n)} & \text { if } 0 \leq i, j<n \\ & \text { otherwise }\end{cases}
$$

Thus,
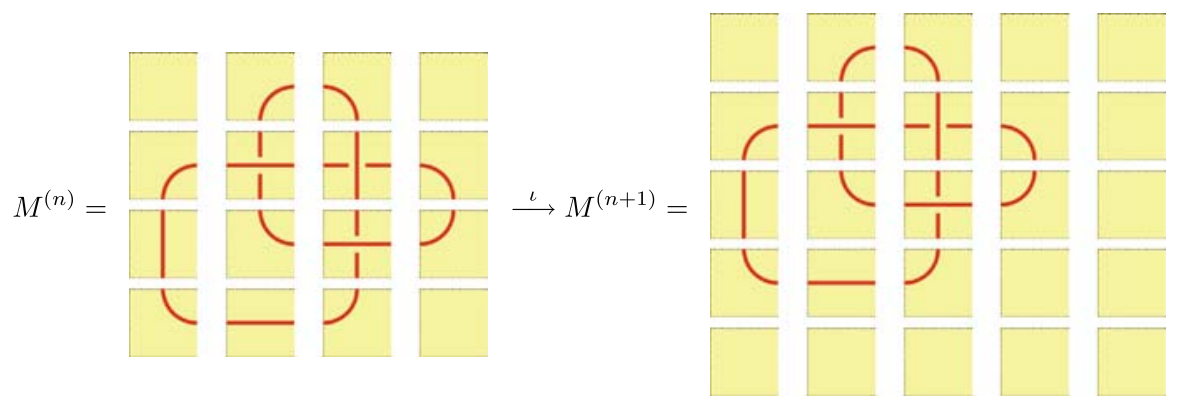

Remark 3 We now can explicitly define the graded system $(\mathbb{K}, \mathbb{A})$ that was mentioned in the introduction. The symbol $\mathbb{K}$ denotes the directed system of sets $\left\{\mathbb{K}^{(n)} \longrightarrow \mathbb{K}^{(n+1)}: n=1,2,3, \ldots\right\}$ and $\mathbb{A}$ denotes the directed system of permutation groups $\{\mathbb{A}(n) \longrightarrow \mathbb{A}(n+1): n=1,2,3, \ldots\}$. Thus,

$$
(\mathbb{K}, \mathbb{A})=\left(\mathbb{K}^{(1)}, \mathbb{A}(1)\right) \longrightarrow\left(\mathbb{K}^{(2)}, \mathbb{A}(2)\right) \longrightarrow \cdots \longrightarrow\left(\mathbb{K}^{(n)}, \mathbb{A}(n)\right) \longrightarrow \cdots
$$

Definition 6 Two $n$-mosaics $M$ and $N$ are said to be of the same knot $n$-type, written

$$
M \underset{n}{\sim} N
$$

provided there is an element of the ambient isotopy group $\mathbb{A}(n)$ which transforms $M$ into $N$.

Definition 7 An $m$-mosaic $M$ and an $n$-mosaic $N$ are said to be of the same knot mosaic type, written

$$
M \sim N
$$


provided there exists a non-negative integer $\ell$ such that, if $m \leq n$, then

$$
\iota^{\ell+n-m} M \sim_{\ell+n} \iota^{\ell} N
$$

or if $m>n$, then

$$
\iota^{\ell} M \sim_{\ell+m} \iota^{\ell+m-n} N,
$$

where, for each non-negative integer $p, \iota^{p}$ denotes the $p$-fold composition $\underbrace{\iota \circ \iota \circ \cdots \circ \iota}_{p}$.

\subsection{Tame knot theory and knot mosaic theory are equivalent}

In the introduction of this paper, we conjecture that the formal (re-writing) system $(\mathbb{K}, \mathbb{A})$ of knot mosaics fully captures the entire structure of tame knot theory. We now explain in greater detail what is meant by this conjecture.

Let $\mathbb{Z}$ denote the set of integers, and $\mathbb{R}^{2}$ the two dimensional Euclidean plane. Let $\tau$ denote the square tiling of $\mathbb{R}^{2}$ induced by the sublattice $\mathbb{Z} \times \mathbb{Z}$ of $\mathbb{R}^{2}$, and for each $i, j$ in $\mathbb{Z}$, let $\tau_{i j}$ denote the subregion of $\mathbb{R}^{2}$ defined by

$$
\tau_{i j}=\left\{(x, y) \in \mathbb{R}^{2}: i \leq x \leq i+1 \text { and } j \leq y \leq j+1\right\} .
$$

Let $k$ be an arbitrary tame knot in 3 -space $\mathbb{R}^{3}$. A knot diagram of $k$, i.e., a regular projection

$$
\pi:\left(\mathbb{R}^{3}, k\right) \longrightarrow\left(\mathbb{R}^{2}, \pi k\right)
$$

is said to be a mosaic knot diagram if

(1) The image under $\pi$ of $k$ lies in the first quadrant of $\mathbb{R}^{2}$, and

(2) For all $i, j$ in $\mathbb{Z}$, the pair $\left(\tau_{i j},(\pi k) \cap \tau_{i j}\right)$ is identical with the cell pair on one of the faces of the 11 tiles $T_{0}, T_{1}, \ldots, T_{10}$.

Remark 4 Clearly, using standard arguments in knot theory, one can prove that every tame knot (or link) has a mosaic knot diagram.

Each mosaic knot diagram $\pi:\left(\mathbb{R}^{3}, k\right) \longrightarrow\left(\mathbb{R}^{2}, \pi k\right)$ of a knot $k$ can naturally be identified with a knot $n$-mosaic $K$, where $n$ is the smallest positive integer such that $\pi k$ lies in the region

$$
\left\{(x, y) \in \mathbb{R}^{2}: 0 \leq x, y \leq n\right\}
$$

Moreover, every knot $n$-mosaic can naturally be identified with the diagram of a knot $k$. We call this associated knot mosaic $K$ a (knot) mosaic representative of the original knot $k$.

This leads us to the following conjecture: 
Conjecture 1 Let $k_{1}$ and $k_{2}$ be two tame knots (or links), and let $K_{1}$ and $K_{2}$ be two arbitrary chosen mosaic representatives of $k_{1}$ and $k_{2}$, respectively. Then $k_{1}$ and $k_{2}$ are of the same knot type if and only if the representative mosaics $K_{1}$ and $K_{2}$ are of the same knot mosaic type. In other words, knot mosaic type is a complete invariant of tame knots.

\section{Part 2: quantum knots}

3.1 Quantum knot systems, quantum knots, and the ambient group $\mathbb{A}$

Our sole purpose in creating the formal system $(\mathbb{K}, \mathbb{A})$ of knot mosaics was to create a framework within which we can explicitly define what is meant by a quantum knot. We are finally in a position to do so.

We begin by assigning a left-to-right linear ordering, denoted by ' $<$ ', to the 11 mosaic tiles as indicated below

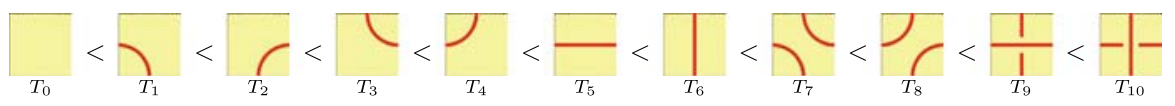

We let $\mathcal{H}$ be the 11 dimensional Hilbert space with orthonormal basis $\left\{\left|T_{p}\right\rangle\right.$ : $0 \leq p<n\}$ labeled by the above mosaic tiles, and we define the Hilbert space $\mathcal{M}^{(n)}$ of $n$-mosaics as the tensor product

$$
\mathcal{M}^{(n)}=\bigotimes_{p=0}^{n^{2}-1} \mathcal{H}
$$

Thus, the induced orthonormal basis of $\mathcal{M}^{(n)}$ consists of all possible $n^{2}$-fold tensor products of the above 11 mosaic tiles, i.e., the induced basis is

$$
\left.\left\{\bigotimes_{p=0}^{n^{2}-1} \mid T_{\ell(p)}\right)\right\} .
$$

We then use the above defined linear ordering on the set $\mathbb{T}^{(n)}$ of mosaic tiles to lexicographically (lex) order all the basis elements of $\mathcal{M}^{(n)}$. We also denote this linear ordering by ' $<$ '. Finally, using row major order, we identify each basis element $n^{2}-1$

$\bigotimes_{p=0}^{n^{2}-1}\left|T_{\ell(p)}\right\rangle$ with the ket $|M\rangle$ labeled by the $n$-mosaic $M=\left(M_{i j}\right)=\left(T_{\ell(n i+j)}\right)$. In other words, we have used row major order to set up a one-to-one correspondence between basis elements of $\mathcal{M}^{(n)}$ and the set of $n$-mosaics $\mathbb{M}^{(n)}$.

For example, for $n=3$ the basis element

$$
\left|T_{2}\right\rangle \otimes\left|T_{5}\right\rangle \otimes\left|T_{4}\right\rangle \otimes\left|T_{9}\right\rangle \otimes\left|T_{2}\right\rangle \otimes\left|T_{1}\right\rangle \otimes\left|T_{5}\right\rangle \otimes\left|T_{8}\right\rangle \otimes\left|T_{3}\right\rangle
$$


is identified with the $n$-mosaic labeled ket

$$
\left|\begin{array}{lll}
T_{2} & T_{5} & T_{4} \\
T_{9} & T_{2} & T_{1} \\
T_{5} & T_{8} & T_{3}
\end{array}\right|
$$

We can now define the Hilbert space of knot n-mosaics $\mathcal{K}^{(n)}$ as the sub-Hilbert space $\mathcal{K}^{(n)}$ of $\mathcal{M}^{(n)}$ spanned by all orthonormal basis elements labeled by knot $n$-mosaics.

Since

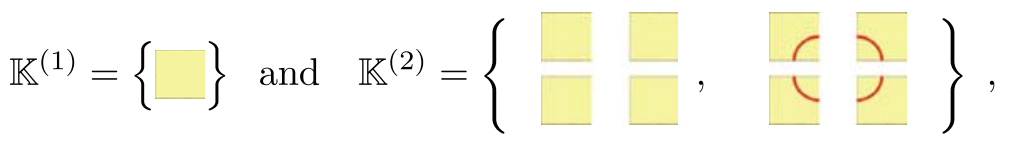

the first two Hilbert spaces $\mathcal{K}^{(1)}$ and $\mathcal{K}^{(2)}$ are of dimensions 1 and 2, respectively. The third Hilbert space $\mathcal{K}^{(3)}$ is of dimension 22, as is demonstrated by the complete list of all possible knot 3-mosaics given Appendix A.

An example of an element of the Hilbert space $\mathcal{K}^{(4)}$ is

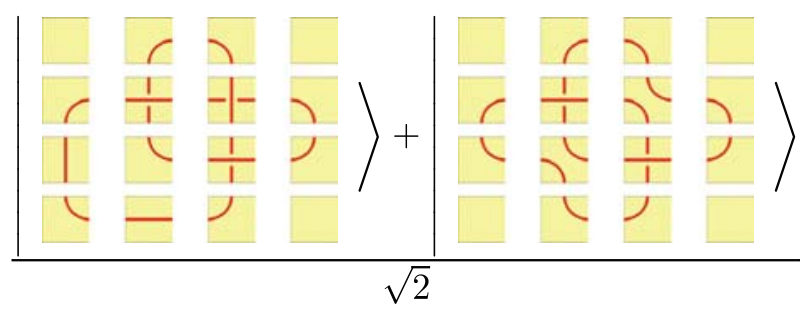

Our next step is to identify each element $g$ of the ambient group $\mathbb{A}(n)$ with the linear transformation

$$
\begin{aligned}
& \mathcal{K}^{(n)} \stackrel{g}{\longrightarrow} \mathcal{K}^{(n)} \\
& |K\rangle \longmapsto|g K\rangle
\end{aligned} .
$$

This is a unitary transformation, since the element $g$ simply permutes the basis elements of $\mathcal{K}^{(n)}$. In this way, the ambient group $\mathbb{A}(n)$ is identified with a discrete unitary subgroup (also denoted by $\mathbb{A}(n)$ ) of the group $U\left(\mathcal{K}^{(n)}\right)$, where $U\left(\mathcal{K}^{(n)}\right)$ denotes the group of all unitary transformations on the Hilbert space $\mathcal{K}^{(n)}$. The unitary subgroup $\mathbb{A}(n)$ will also be called the ambient group.

Finally, everything comes together with the following definition:

Definition 8 Let $n$ be a positive integer. A quantum knot system $Q\left(\mathcal{K}^{(n)}, \mathbb{A}(n)\right)$ of order $n$ is a quantum system with the Hilbert space $\mathcal{K}^{(n)}$ of knot $n$-mosaics as its state space, and having the ambient group $\mathbb{A}(n)$ as an accessible unitary control group. The states of the quantum system $Q\left(\mathcal{K}^{(n)}, \mathbb{A}(n)\right)$ are called quantum knots of order $n$, and the elements of the ambient group $\mathbb{A}(n)$ are called unitary knot moves. Moreover, the quantum knot system $Q\left(\mathcal{K}^{(n)}, \mathbb{A}(n)\right)$ of order $n$ is a subsystem of the quantum knot system $Q\left(\mathcal{K}^{(n+1)}, \mathbb{A}(n+1)\right)$ of order $n+1$. Thus, the quantum knot systems 
$Q\left(\mathcal{K}^{(n)}, \mathbb{A}(n)\right)$ collectively become a nested sequence of quantum knot systems which we will denote simply by $Q(\mathcal{K}, \mathbb{A})$. In other words,

$$
\begin{aligned}
Q(\mathcal{K}, \mathbb{A})= & Q\left(\mathcal{K}^{(1)}, \mathbb{A}(1)\right) \longrightarrow Q\left(\mathcal{K}^{(2)}, \mathbb{A}(2)\right) \longrightarrow \cdots \longrightarrow \\
& Q\left(\mathcal{K}^{(n)}, \mathbb{A}(n)\right) \longrightarrow \cdots
\end{aligned}
$$

Remark 5 The nested quantum knot system $Q(\mathcal{K}, \mathbb{A})$ is probably not a physically realizable system. However, each quantum knot system $Q\left(\mathcal{K}^{(n)}, \mathbb{A}(n)\right)$ of order $n$ is physically realizable. ${ }^{5}$

Example 1 As an example, in the quantum system $Q\left(\mathcal{K}^{(5)}, \mathbb{A}(5)\right)$, we see that the action of the unitary Reidemeister 2 move

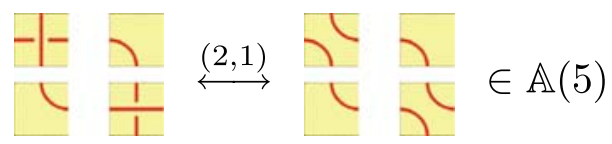

on the quantum knot

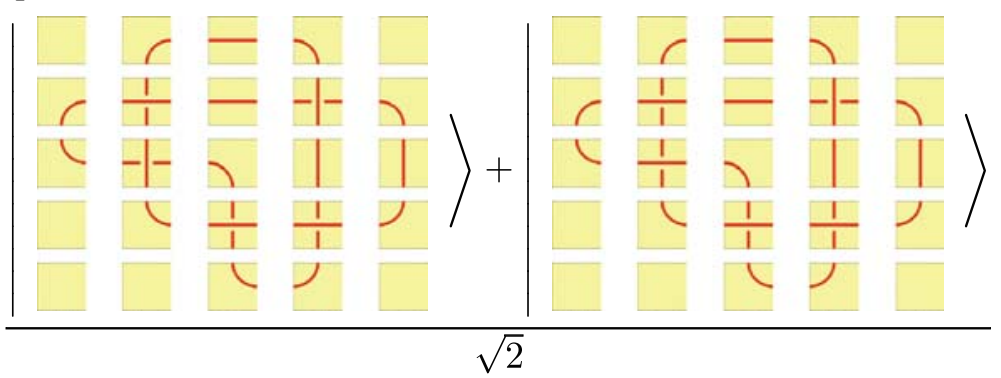

produces the quantum knot

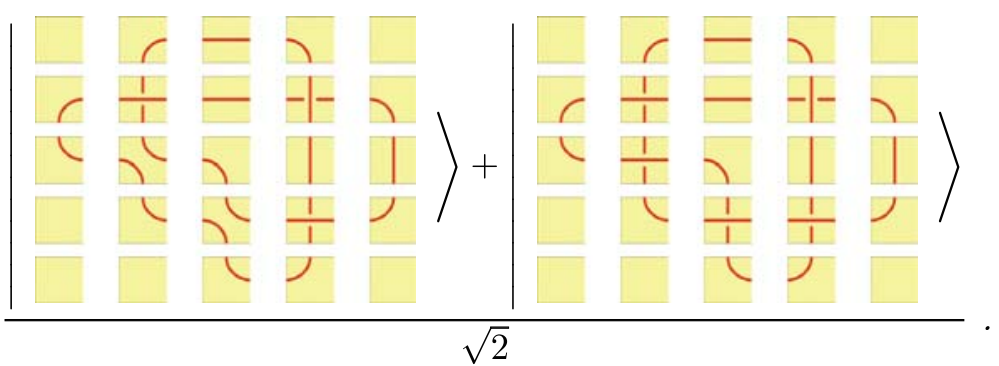

\subsection{Quantum knot type}

When are two quantum knots the same?

\footnotetext{
5 It should be mentioned that, although the quantum knot system $Q\left(\mathcal{K}^{(n)}, \mathbb{A}(n)\right)$ is physically realizable, it may or may not be implentable within today's existing technology.
} 
Definition 9 Let $\left|\psi_{1}\right\rangle$ and $\left|\psi_{2}\right\rangle$ be two quantum knots of a quantum system $Q\left(\mathcal{K}^{(n)}\right.$, $\mathbb{A}(n))$ of order $n$. Then $\left|\psi_{1}\right\rangle$ and $\left|\psi_{2}\right\rangle$ are said to be of the same quantum knot n-type, written

$$
\left|\psi_{1}\right\rangle \underset{n}{\sim}\left|\psi_{2}\right\rangle
$$

provided there exists a unitary transformation $g$ in the ambient group $\mathbb{A}(n)$ which transforms $\left|\psi_{1}\right\rangle$ into $\left|\psi_{2}\right\rangle$, i.e., such that

$$
g\left|\psi_{1}\right\rangle=\left|\psi_{2}\right\rangle
$$

They are said to be of the same quantum knot type, written

$$
\left|\psi_{1}\right\rangle \sim\left|\psi_{2}\right\rangle
$$

provided that for some non-negative integer $\ell$,

$$
\iota^{\ell}\left|\psi_{1}\right\rangle \underset{n+\ell}{\sim} \iota^{\ell}\left|\psi_{2}\right\rangle,
$$

where $\iota^{\ell}\left|\psi_{1}\right\rangle$ and $\iota^{\ell}\left|\psi_{2}\right\rangle$ are states of the quantum system $Q\left(\mathcal{K}^{(n+\ell)}, \mathbb{A}(n+\ell)\right)$, where $\iota: \mathcal{K}^{(m)} \longrightarrow \mathcal{K}^{(m+1)}$ is the monomorphism induced by the previously defined injection $\iota: \mathbb{K}^{(m)} \longrightarrow \mathbb{K}^{(m+1)}$.

Thus, the two quantum knots found in the last example of the previous section are of the same quantum knot 5-type, and also of the same quantum knot type. Surprisingly, the following two quantum knots $\left|\psi_{1}\right\rangle$ and $\left|\psi_{2}\right\rangle$ are neither of the same quantum knot 3-type nor knot type:

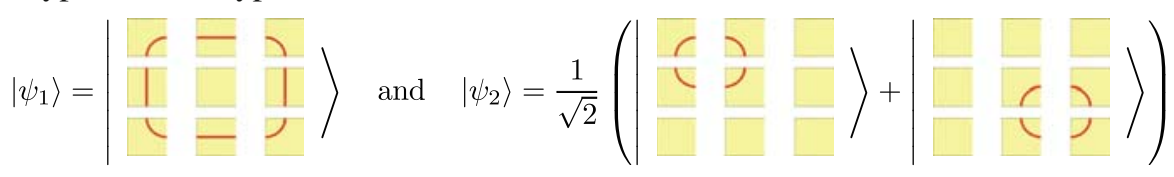

This follows from the fact that the ambient group $\mathbb{A}(n)$ is generated by a finite set of involutions.

\subsection{Hamiltonians of the generators of the ambient group $\mathbb{A}$}

In this section, we show how to find the Hamiltonians associated with the generators of the ambient group $\mathbb{A}(n)$, i.e., the planar isotopy and Reidemeister moves for quantum knots.

Let $g$ be an arbitrary planar isotopy move or Reidemeister move in the ambient group $\mathbb{A}(n)$. From proposition 1, we know that $g$, as a permutation, is the product of disjoint transpositions of knot $n$-mosaics, i.e., of the form

$$
g=\left(K_{\alpha_{1}}, K_{\beta_{1}}\right)\left(K_{\alpha_{2}}, K_{\beta_{2}}\right) \cdots\left(K_{\alpha_{\ell}}, K_{\beta_{\ell}}\right)
$$


Without loss of generality, we can assume that $\alpha_{j}<\beta_{j}$ for $1 \leq j \leq \ell$, and that $\alpha_{j}<\alpha_{j+1}$ for $1 \leq j<\ell$, where ' $<$ ' denotes the previously defined lexicographic (lex) order on the set of $n$-mosaics $\mathbb{M}^{(n)}$. For each permutation $\eta$ of $\mathbb{M}^{(n)}$, let ' $<_{\eta}$ ' denote the new linear ordering created by the application of the permutation $\eta$.

Choose a permutation $\eta$ such that

$$
K_{\alpha_{1}}<_{\eta} K_{\beta_{1}}<_{\eta} K_{\alpha_{2}}<_{\eta} K_{\beta_{2}}<_{\eta} \cdots<_{\eta} K_{\alpha_{\ell}}<_{\eta} K_{\beta_{\ell}}
$$

with $K_{\beta_{\ell}}<_{\eta}$ all other $n$-mosaics, and let $\sigma_{0}$ and $\sigma_{1}$ denote respectively the identity matrix and the first Pauli spin matrix given below

$$
\sigma_{0}=\left(\begin{array}{ll}
1 & 0 \\
0 & 1
\end{array}\right) \text { and } \sigma_{1}=\left(\begin{array}{ll}
0 & 1 \\
1 & 0
\end{array}\right)
$$

Then in the $\eta$-reordered basis of the Hilbert space $\mathcal{K}^{(n)}$, the element $g$, as a unitary transformation, is of the form

$$
\eta^{-1} g \eta=\left(\begin{array}{ccccc}
\sigma_{1} & O & \ldots & O & O \\
O & \sigma_{1} & \ldots & O & O \\
\vdots & \vdots & \ddots & & \vdots \\
O & O & \ldots & \sigma_{1} & O \\
O & O & \ldots & O & I_{n-2 \ell}
\end{array}\right)=\left(I_{\ell} \otimes \sigma_{1}\right) \oplus I_{n-2 \ell}
$$

where ' $O$ ' denotes an all zero matrix of appropriate size, where $I_{n-2 \ell}$ denotes the $(n-2 \ell) \times(n-2 \ell)$ identity matrix, and where ' $\oplus$ ' denotes the direct sum of matrices, i.e., $A \oplus B=\left(\begin{array}{cc}A & O \\ O & B\end{array}\right)$.

The natural $\log$ of $\sigma_{1}$ is

$$
\ln \sigma_{1}=\frac{i \pi}{2}(2 s+1)\left(\sigma_{0}-\sigma_{1}\right)
$$

where $s$ denotes an arbitrary integer. Hence, the natural $\log , \ln \left(\eta^{-1} g \eta\right)$, of the unitary transformation $\eta^{-1} g \eta$ is

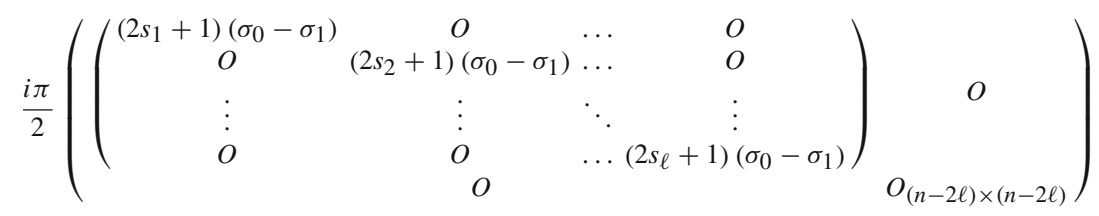

where $s_{1}, s_{2}, \ldots, s_{\ell}$ are arbitrary integers. ${ }^{6}$

\footnotetext{
$\overline{6 \text { Let } U \text { be an arbitrary finite } r} \times r$ unitary matrix, and let $W$ be a unitary matrix that diagonalizes $U$, i.e., a unitary matrix $W$ such that $W U W^{-1}=\Delta(\lambda(1), \lambda(2) \ldots, \lambda(r))$. Then the natural $\log$ of $A$ is $\ln A=W^{-1} \Delta(\ln \lambda(1), \ln \lambda(2) \ldots, \ln \lambda(r)) W$.
} 
Since we are interested only in the simplest Hamiltonian, we choose the principal branch $\ln _{P}$ of the natural $\log$, i.e., the branch for which $s_{1}=s_{2}=\cdots s_{\ell}=0$, and obtain for our Hamiltonian

$$
H_{g}=-i \eta\left[\ln P\left(\eta^{-1} g \eta\right)\right] \eta^{-1}=\frac{\pi}{2} \eta\left(\begin{array}{cc}
I_{\ell} \otimes\left(\sigma_{0}-\sigma_{1}\right) & O \\
O & O_{(n-2 \ell) \times(n-2 \ell)}
\end{array}\right) \eta^{-1}
$$

Thus, if the initial quantum knot is

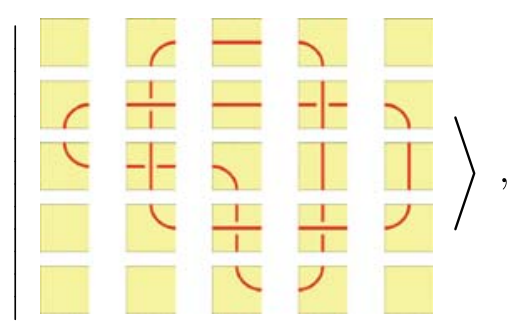

and if we use the Hamiltonian $H_{g}$ for the Reidemeister 2 move

$$
g=\frac{-1-}{5} \frac{1}{\frac{1}{1}} \stackrel{(2,1)}{\longleftrightarrow} \frac{\sqrt{5}}{\sqrt{1}},
$$

then the solution to Schroedinger's equation is

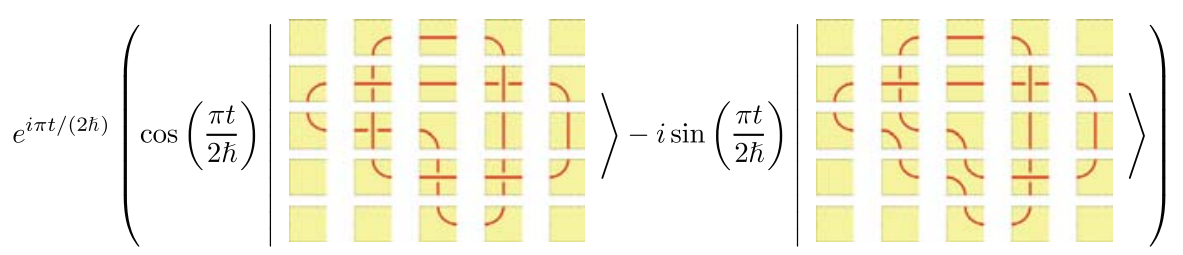

where $t$ denotes time, and where $\hbar$ denotes Planck's constant divided by $2 \pi$.

\subsection{Knot crossing tunnelling and other unitary transformations}

We should also mention a number of other miscellaneous unitary transformations that do not lie in the ambient group $\mathbb{A}(n)$, but are nonetheless of interest. by

There is the unitary transformation $\tau_{i j}$, called the tunnelling transformation, given

$$
\tau_{i j}=\frac{1}{1} \stackrel{(i, j)}{\longleftrightarrow}-\mid-
$$

which enables a quantum knot overcrossing to tunnel into an undercrossing, or vice versa.

From this, we can construct the mirror image transformation given by 


$$
\mu=\prod_{i, j=0}^{n-1}\left(\frac{1}{1} \stackrel{(i, j)}{\longleftrightarrow}-\mid-\right)
$$

that transforms a quantum knot into its mirror image.

The following two unitary transformations can be used to create four dimensional quantum knots. ${ }^{7}$ The first is the hyperbolic transformation $\eta_{i j}$ given by

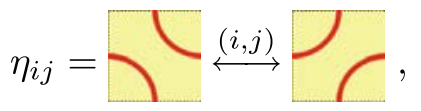

and the second is the elliptic transformation $\varepsilon_{i j}$ by

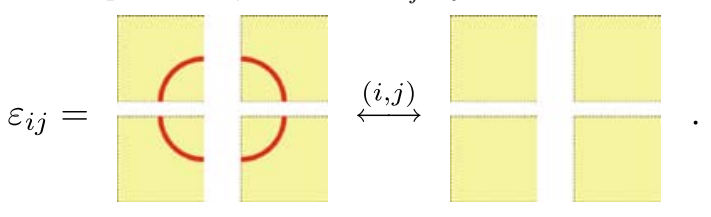

More will be said about these transformations in future papers.

\subsection{Quantum observables as invariants of quantum knots}

We now consider the following question:

Question: What is a quantum knot invariant? How do we define it?

The objective of the first half of this section is to give a discursive argument that justifies a definition which will be found to be equivalent to the following:

A quantum knot n-invariant for a quantum system $Q\left(\mathcal{K}^{(n)}, \mathbb{A}(n)\right)$ is an observable $\Omega$ on the Hilbert space $\mathcal{K}^{(n)}$ of quantum knots which is invariant under the action of the ambient group $\mathbb{A}(n)$, i.e., such that $U \Omega U^{-1}=\Omega$ for all $U$ in $\mathbb{A}(n)$.

Caveat: We emphasize to the reader that the above definition of quantum knot invariants is not the one currently used in quantum topology. Quantum topology uses analogies with quantum mechanics to create significant mathematical structures that do not necessarily correspond directly to quantum mechanical observables. The invariants of quantum topology have been investigated for their relevance to quantum computing and they can be regarded, in our context, as possible secondary calculations made on the basis of an observable. Here we are concerned with observables that are themselves topological invariants.

To justify our new use of the term 'quantum knot invariant,' we will use the following YARDSTICK:

YARDSTICK: Quantum knot invariants are to be physically meaningful invariants of quantum knot type. By "physically meaningful," we mean that the quantum knot invariants can be directly obtained from experimental data produced by an implementable physical experiment. ${ }^{8}$

\footnotetext{
7 A four dimensional classical knot is a knotted 2-sphere in 4-space. For readers interested in learning more about higher dimensional knot theory, we refer them to [18] and [19].

8 Once again we remind the reader that, although the quantum $\operatorname{knot}$ system $Q\left(\mathcal{K}^{(n)}, \mathbb{A}(n)\right)$ is physically implementable, it may or may not be implentable within today's existing technology.
} 
Let $Q\left(\mathcal{K}^{(n)}, \mathbb{A}(n)\right)$ be a quantum knot system, where $\mathcal{K}^{(n)}$ is the Hilbert space of quantum knots, and where $\mathbb{A}(n)$ is the underlying ambient group on $\mathcal{K}^{(n)}$. Moreover, let $\mathcal{P}^{(n)}$ denote some yet-to-be-chosen mathematical domain. By an $n$-invariant $I^{(n)}$ of quantum knots, we mean a map

$$
I^{(n)}: \mathcal{K}^{(n)} \longrightarrow \mathcal{P}^{(n)},
$$

such that, when two quantum knots $\left|\psi_{1}\right\rangle$ and $\left|\psi_{2}\right\rangle$ are of the same knot $n$-type, i.e., when

$$
\left|\psi_{1}\right\rangle \underset{n}{\sim}\left|\psi_{2}\right\rangle
$$

then their respective invariants must be equal, i.e.,

$$
I^{(n)}\left(\left|\psi_{1}\right\rangle\right)=I^{(n)}\left(\left|\psi_{2}\right\rangle\right)
$$

In other words, $I^{(n)}: \mathcal{K}^{(n)} \longrightarrow \mathcal{P}^{(n)}$ is a map which is invariant under the action of the ambient group $\mathbb{A}(n)$, i.e.,

$$
I^{(n)}(|\psi\rangle)=I^{(n)}(g|\psi\rangle)
$$

for all elements $g$ in $\mathbb{A}(n)$.

Question: But which such invariants are physically meaningful?

We begin to try to answer this question by noting that the only way to extract information from a quantum system is through quantum measurement. Thus, if we wish to extract information about quantum knot type from a quantum knot system $Q\left(\mathcal{K}^{(n)}, \mathbb{A}(n)\right)$,we of necessity must make a measurement with respect to some observable. But what kind of observable?

With this in mind, we will now describe quantum measurement from a different, but nonetheless equivalent perspective, than that which is usually given in standard texts on quantum mechanics. ${ }^{9}$ For knot theorists who might not be familiar with standard quantum measurement, we have included in the figure below a brief summary of quantum measurement. ${ }^{10}$

\footnotetext{
${ }^{9}$ In this paper, we will focus only on von Neumann quantum measurement. We will discuss more the general POVM approach to quantum knot invariants in a later paper.

${ }^{10}$ For readers unfamiliar with quantum measurement, there are many references, for example, $[2,5,16,17$, $21,22,24,25]$.
} 


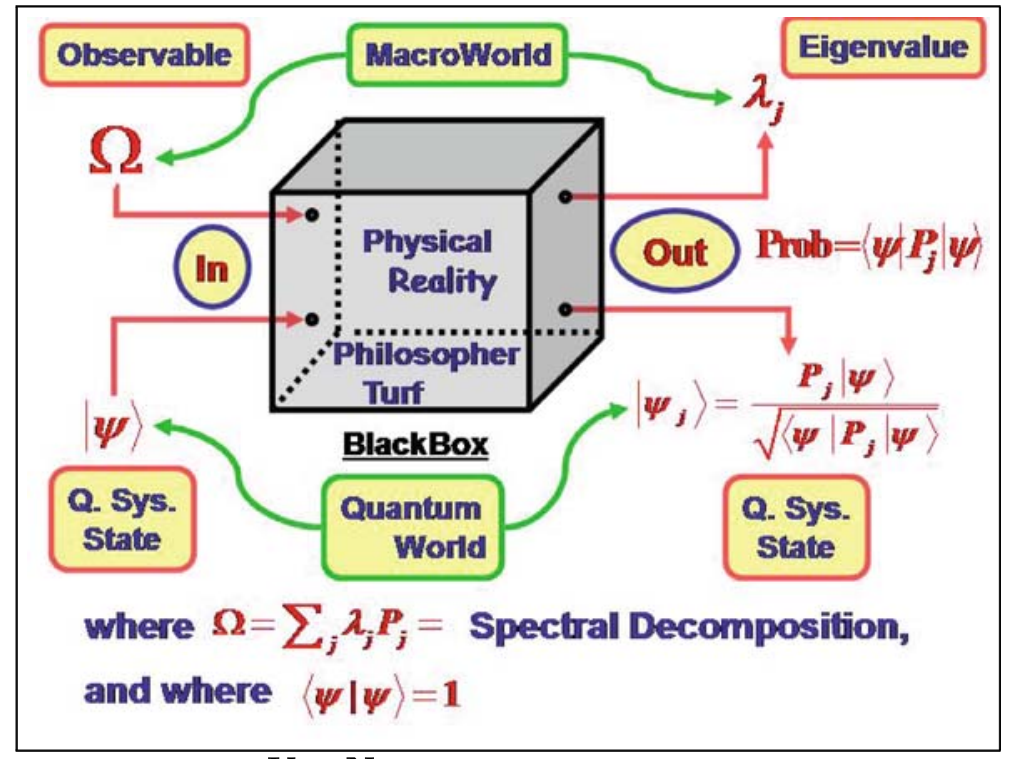

\section{Von Neumann measurement.}

Let $\Omega$ be an observable for a quantum system $Q\left(\mathcal{K}^{(n)}, \mathbb{A}(n)\right)$, i.e., a Hermitian (self-adjoint) linear operator on the Hilbert space $\mathcal{K}^{(n)}$. Moreover, let

$$
\Omega=\sum_{j=1}^{m} \lambda_{j} P_{j}
$$

be the spectral decomposition of the observable $\Omega$, where $\lambda_{j}$ is the $j$-th eigenvalue of $\Omega$, and where $P_{j}$ is the corresponding projection operator for the associated eigenspace $V_{j}$.

Let $\mathcal{P}_{\Omega}^{(n)}$ denote the set of all probability distributions on the spectrum $\left\{\lambda_{1}, \lambda_{2}, \ldots, \lambda_{m}\right\}$ of $\Omega$, i.e.,

$$
\mathcal{P}_{\Omega}^{(n)}=\left\{p:\left\{\lambda_{1}, \lambda_{2}, \ldots, \lambda_{m}\right\} \longrightarrow[0,1]: \sum_{j=1}^{m} p\left(\lambda_{j}\right)=1\right\}
$$

We will call the probability distributions $p$ of $\mathcal{P}_{\Omega}^{(n)}$ stochastic sources.

Then each observable $\Omega$ uniquely determines a map

$$
\begin{aligned}
\widetilde{\Omega}: \mathcal{K}^{(n)} & \longrightarrow \mathcal{P}_{\Omega}^{(n)} \\
|\psi\rangle & \longmapsto p
\end{aligned}
$$

from quantum knots to stochastic sources on the spectrum of $\Omega$ given by

$$
p_{j}(|\psi\rangle)=\frac{\left\langle\psi\left|P_{j}\right| \psi\right\rangle}{\sqrt{\langle\psi \mid \psi\rangle}}
$$


Thus, what is seen, when a quantum system $Q$ in state $|\psi\rangle$ is measured with respect to an observable $\Omega$, is a random sample from the stochastic source $\widetilde{\Omega}(|\psi\rangle)$. But under what circumstances is such a random sample a quantum knot invariant?

Our answer to this question is that quantum knots $\left|\psi_{1}\right\rangle$ and $\left|\psi_{2}\right\rangle$ of the same knot $n$-type must produce random samples from the same stochastic source when measured with respect to the observable $\Omega$. This answer is captured by the following definition:

Definition 10 Let $Q\left(\mathcal{K}^{(n)}, \mathbb{A}(n)\right)$ be a quantum knot system, and let $\Omega$ be an observable on $\mathcal{K}^{(n)}$ with spectral decomposition

$$
\Omega=\sum_{j=1}^{m} \lambda_{j} P_{j} .
$$

Then the observable $\Omega$ is said to be a quantum knot $n$-invariant provided

$$
\left\langle\psi\left|U P_{j} U^{-1}\right| \psi\right\rangle=\left\langle\psi\left|P_{j}\right| \psi\right\rangle
$$

for all quantum knots $|\psi\rangle \in \mathcal{K}^{(n)}$, for all $U \in \mathbb{A}(n)$, and for all projectors $P_{j}$.

Theorem 1 Let $Q\left(\mathcal{K}^{(n)}, \mathbb{A}(n)\right)$ and $\Omega$ be as given in the above definition. Then the following statements are equivalent:

(1) The observable $\Omega$ is a quantum knot n-invariant

(2) $\left[U, P_{j}\right]=0$ for all $U \in \mathbb{A}(n)$ and for all $P_{j}$.

(3) $[U, \Omega]=0$ for all $U \in \mathbb{A}(n)$,

where $[A, B]$ denotes the commutator $A B-B A$ of operators $A$ and $B$.

The remaining half of this section is devoted to finding an answer to the following question:

Question: How do we find observables which are quantum knot invariants?

One answer to this question is the following theorem, which is an almost immediate consequence of the definition of a minimum invariant subspace of $\mathcal{K}^{(n)}$ :

Theorem 2 Let $Q\left(\mathcal{K}^{(n)}, \mathbb{A}(n)\right)$ be a quantum knot system, and let

$$
\mathcal{K}^{(n)}=\bigoplus_{\ell} W_{\ell}
$$

be a decomposition of the representation

$$
\mathbb{A}(n) \times \mathcal{K}^{(n)} \longrightarrow \mathcal{K}^{(n)}
$$

into irreducible representations of the ambient group $\mathbb{A}(n)$. Then, for each $\ell$, the projection operator $P_{\ell}$ for the subspace $W_{\ell}$ is an observable which is a quantum knot n-invariant. 
Here is yet another way of finding quantum knot invariants:

Theorem 3 Let $Q\left(\mathcal{K}^{(n)}, \mathbb{A}(n)\right)$ be a quantum knot system, and let $\Omega$ be an observable on the Hilbert space $\mathcal{K}^{(n)}$. Let $S t(\Omega)$ be the stabilizer subgroup for $\Omega$, i.e.,

$$
S t(\Omega)=\left\{U \in \mathbb{A}(n): U \Omega U^{-1}=\Omega\right\} \text {. }
$$

Then the observable

$$
\sum_{U \in \mathbb{A}(n) / S t(\Omega)} U \Omega U^{-1}
$$

is a quantum knot $n$-invariant, where $\sum_{U \in \mathbb{A}(n) / S t(\Omega)} U \Omega U^{-1}$ denotes a sum over a complete set of coset representatives for the stabilizer subgroup $S t(\Omega)$ of the ambient group $\mathbb{A}(n)$.

Proof The observable $\sum_{g \in \mathbb{A}(n)} g \Omega g^{-1}$ is obviously an quantum knot $n$-invariant, since $g^{\prime}\left(\sum_{g \in \mathbb{A}(n)} g \Omega g^{-1}\right) g^{\prime-1}=\sum_{g \in \mathbb{A}(n)} g \Omega g^{-1}$ for all $g^{\prime} \in \mathbb{A}(n)$. If we let $|S t(\Omega)|$ denote the order of $|S t(\Omega)|$, and if we let $c_{1}, c_{2}, \ldots, c_{p}$ denote a complete set of coset representatives of the stabilizer subgroup $S t(\Omega)$, then $\sum_{j=1}^{p} c_{j} \Omega c_{j}^{-1}=\frac{1}{|\operatorname{St}(\Omega)|}$ $\sum_{g \in \mathbb{A}(n)} g \Omega g^{-1}$ is also a quantum knot invariant.

We end this section with an example of a quantum knot invariant:

Example 2 The following observable $\Omega$ is an example of a quantum knot 4-invariant:

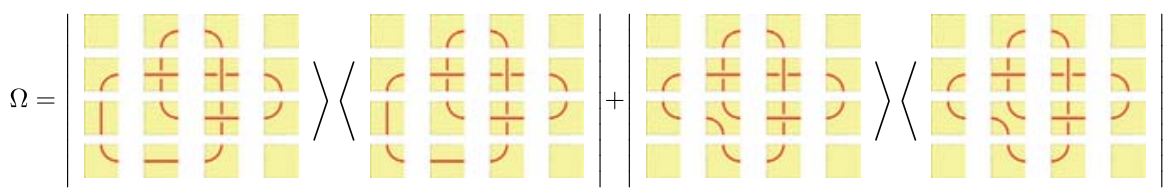

Remark 6 For yet another approach to quantum knot measurement, we refer the reader to the brief discussion on quantum knot tomography found in item (11) in the conclusion of this paper.

\section{Conclusion: Open questions and future directions}

There are many possible open questions and future directions for research. We mention only a few.

(1) What is the exact structure of the ambient group $\mathbb{A}(n)$ and its direct limit

$$
\mathbb{A}=\lim _{\longrightarrow} \mathbb{A}(n) .
$$

Can one write down an explicit presentation for $\mathbb{A}(n)$ ? for $\mathbb{A}$ ? The fact that the ambient group $\mathbb{A}(n)$ is generated by involutions suggests that it may be a Coxeter group. Is it a Coxeter group? 
(2) Unlike classical knots, quantum knots can exhibit the non-classical behavior of quantum superposition and quantum entanglement. Are topological entanglement and quantum entanglement related to one another? If so, how?

(3) What other ways are there to distinguish quantum knots from classical knots?

(4) How does one find a quantum observable for the Jones polynomial? This would be a family of observables parameterized by points on the unit circle in the complex plane. Does this approach lead to an algorithmic improvement to the quantum algorithm given by Aharonov et al. [1]? (See also $[15,27]$.)

(5) How does one create quantum knot observables that represent other knot invariants such as, for example, the Vassiliev invariants?

(6) What is gained by extending the definition of quantum knot observables to POVMs?

(7) What is gained by extending the definition of quantum knots to mixed ensembles?

(8) Define the mosaic number of a knot $k$ as the smallest integer $n$ for which $k$ is representable as a knot $n$-mosaic. For example, the mosaic number of the trefoil is 4 , as is illustrated by the following knot $n$-mosaic:

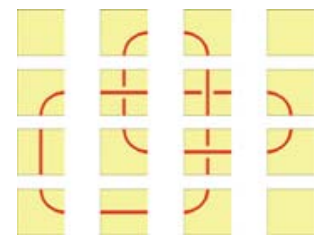

In general, how does one compute the mosaic number? Is the mosaic number related to the crossing number of a knot? How does one find an observable for the mosaic number?

(9) Let $D_{n}$ denote the dimension of the Hilbert space $\mathcal{K}^{(n)}$ of quantum knot $n$-mosaics. We have shown that $D_{1}=1, D_{2}=2$, and $D_{3}=22$. Find $D_{n}$ for other values of $n$. A very loose upper bound for $D_{n}$ is obviously $11^{n^{2}}$.

(10) Consider the following alternate stronger definitions of quantum knot $n$-type and quantum knot type:

Let $Q\left(\mathcal{K}^{(n)}, \mathbb{A}(n)\right)$ be a quantum knot system, and let $\mathcal{U}\left(\mathcal{K}^{(n)}\right)$ denote the Lie group of all unitary transformations on the Hilbert space $\mathcal{K}^{(n)}$. Define the continuous ambient group $\widetilde{\mathbb{A}}(n)$ as the smallest connected Lie subgroup of $\mathcal{U}\left(\mathcal{K}^{(n)}\right)$ containing the discrete ambient group $\mathbb{A}(n)$.

Proposition 2 Let $\mathcal{G}$ denote the set of planar isotopy and Reidemeister generators of the discrete ambient group $\mathbb{A}(n)$, and let $a(n)$ be the Lie algebra generated by the elements of the set

$$
\left\{\ln _{P}(g): g \in \mathcal{G}\right\}
$$

where $\ln _{P}$ denotes the principal branch of the natural log on $\mathcal{U}\left(\mathcal{K}^{(n)}\right)$. Then the continuous ambient group is given by

$$
\widetilde{\mathbb{A}}(n)=\exp (a(n))
$$


We define two quantum $n$-knots $\left|\psi_{1}\right\rangle$ and $\left|\psi_{2}\right\rangle$ to be of the same continuous knot n-type, written

$$
\left|\psi_{2}\right\rangle \underset{n}{\approx}\left|\psi_{2}\right\rangle
$$

provided there exists an element $g$ of the continuous ambient group $\widetilde{\mathbb{A}}(n)$ which transforms $\left|\psi_{1}\right\rangle$ into $\left|\psi_{2}\right\rangle$, i.e., such that $g\left|\psi_{1}\right\rangle=\left|\psi_{2}\right\rangle$. They are of the same continuous knot type, written $\left|\psi_{1}\right\rangle \approx\left|\psi_{2}\right\rangle$, if there exists an integer $\ell$ such that $\iota^{\ell}\left|\psi_{1}\right\rangle \underset{n+\ell}{\approx} \iota^{\ell}\left|\psi_{2}\right\rangle$.

Conjecture 2 Let $K_{1}$ and $K_{2}$ denote two knot n-mosaics, and let $\left|K_{1}\right\rangle$ and $\left|K_{2}\right\rangle$ denote the corresponding quantum knots. Then

$$
\left|K_{1}\right\rangle \underset{n}{\approx}\left|K_{2}\right\rangle \Longleftrightarrow K_{1} \underset{n}{\sim} K_{2} \text { and }\left|K_{1}\right\rangle \approx\left|K_{2}\right\rangle \Longleftrightarrow K_{1} \sim K_{2}
$$

Thus, if Conjectures 1 and 2 are true, these two stronger definitions of quantum knot $n$-type and quantum knot type fully capture all of classical tame knot theory. Moreover, these two stronger definitions have a number of advantages over the weaker definitions, two of which are the following:

- Under the Hamiltonians associated with the generators $\mathcal{G}$, the Schroedinger equation determines a connected continuous path in $\mathcal{K}^{(n)}$ consisting of quantum $n$-knots all of the same quantum continuous knot $n$-type.

- Although the following two quantum knots are not of the same discrete knot $n$-type, they are however of the same continuous knot $n$-type

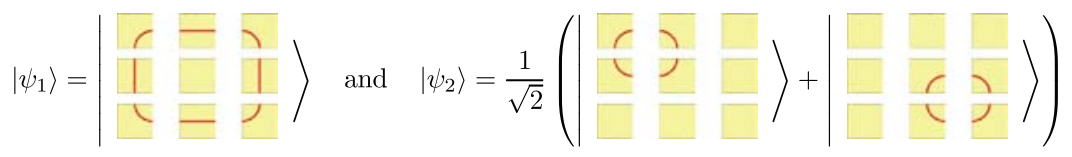

Is the above conjecture true? If so, what is the structure of the continuous ambient group $\widetilde{\mathbb{A}}(n)$ ? What are its irreducible representations?

(10a) Can one create a more continuous definition of a quantum knot by quantizing the classical electromagnetic knots found in [14]? This question was the original motivation for this paper.

(11) Quantum knot tomography: Given repeated copies of a quantum knot $|\psi\rangle$, how does one employ the method of quantum state tomography [12] to determine $|\psi\rangle$ ? Most importantly, how can this be done with the greatest efficiency? For example, given repeated copies of the unknown quantum knot basis state

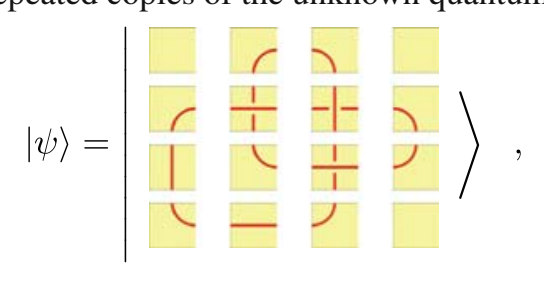


one could make repeated measurements of this state with respect to the $11 \cdot n^{2}$ (for $n=4$ ) tile observables

$$
\left\{\Omega_{i j}^{(p)}=1^{\otimes(n j+i-1)} \otimes\left(\left|T_{p}\right\rangle\left\langle T_{p}\right|\right) \otimes 1^{\otimes\left(n^{2}-n j-i\right)}: 0 \leq p<11,0 \leq i, j<n\right\}
$$

to determine the state with a probability of error less than a chosen positive threshold $\epsilon$. This is obviously not the most efficient set of observables for the task, nor is it a universal set of observables for quantum knot tomography. Given many copies of an arbitrary quantum knot, how does find a universal set of observables that is best in the sense of greatest efficiency for a given threshold $\epsilon$ ?

(12) Quantum Braids: One can also use mosaics to define quantum braids. How is this related to the work found in $[6,11,26]$ ?

(13) Can quantum knot systems be used to model and to predict the behavior of

(i) Quantum vortices in liquid Helium II? (See [23].)

(ii) Quantum vortices in the Bose-Einstein condensate? (See [30].)

(iii) Fractional charge quantification that is manifest in the fractional quantum Hall effect? (See [6] and [28].)

We should mention that we have throughout this paper used a version of knot diagrams (i.e., two dimensional knot mosaics), that is susceptible to quantization. Within this context, the familiar Reidemeister moves have become unitary transformations on an appropriate Hilbert space. Knots as we know them in topology and geometry occur as embeddings in three dimensional space, and are projected to knot diagrams for combinatorial and topological purposes. Thus, we have chosen to model quantum knots through the extra structure of knot diagrams. However, it is also possible to investigate quantum knots in full three dimensional space by using three dimensional knot mosaics. This will be the subject of a forthcoming paper.

In closing this section, we should finally also say that, in the open literature, the phrase "quantum knot" has many different meanings, and is sometimes a phrase that is used loosely. We mention only two examples. In [7], a quantum knot is essentially defined as an element of the Hilbert space with orthonormal basis in one-one-correspondence with knot types, rather than knot representatives. Within the context of the mosaic construction, a quantum knot in [7] corresponds to an element of the orbit Hilbert space $\mathcal{K}^{(n)} / \mathbb{A}(n)$. In [3] and in [26] the phrase "quantum knot" refers not to knots, but to the use of representations of the braid group to model the dynamic behavior of certain quantum systems. In this contexts, braids are used as a tool to model topological obstructions to quantum decoherence that are conjectured to exist within certain quantum systems. ${ }^{11}$

Acknowledgments The first author would like to thank the Institute for Scientific Interchange (ISI) in Torino, Italy, and the Mathematical Sciences Research Institute in Berkeley, California for providing a research climate where many of the ideas found within this paper could germinate. This effort was partially supported by the Defense Advanced Research Projects Agency (DARPA) and Air Force Research Laboratory, Air Force Materiel Command, USAF, under agreement number F30602-01-2-0522, and by L-OO-P Fund Grant BECA2002. The second author would like to thank the National Science Foundation for support under NSF grant DMS-0245588.

11 See also references [8,9]. 


\section{Appendix A: A list of all knot 3-mosaics}

A complete list of all knot 3-mosaics, listed in lexicographic (lex) order, is given below:
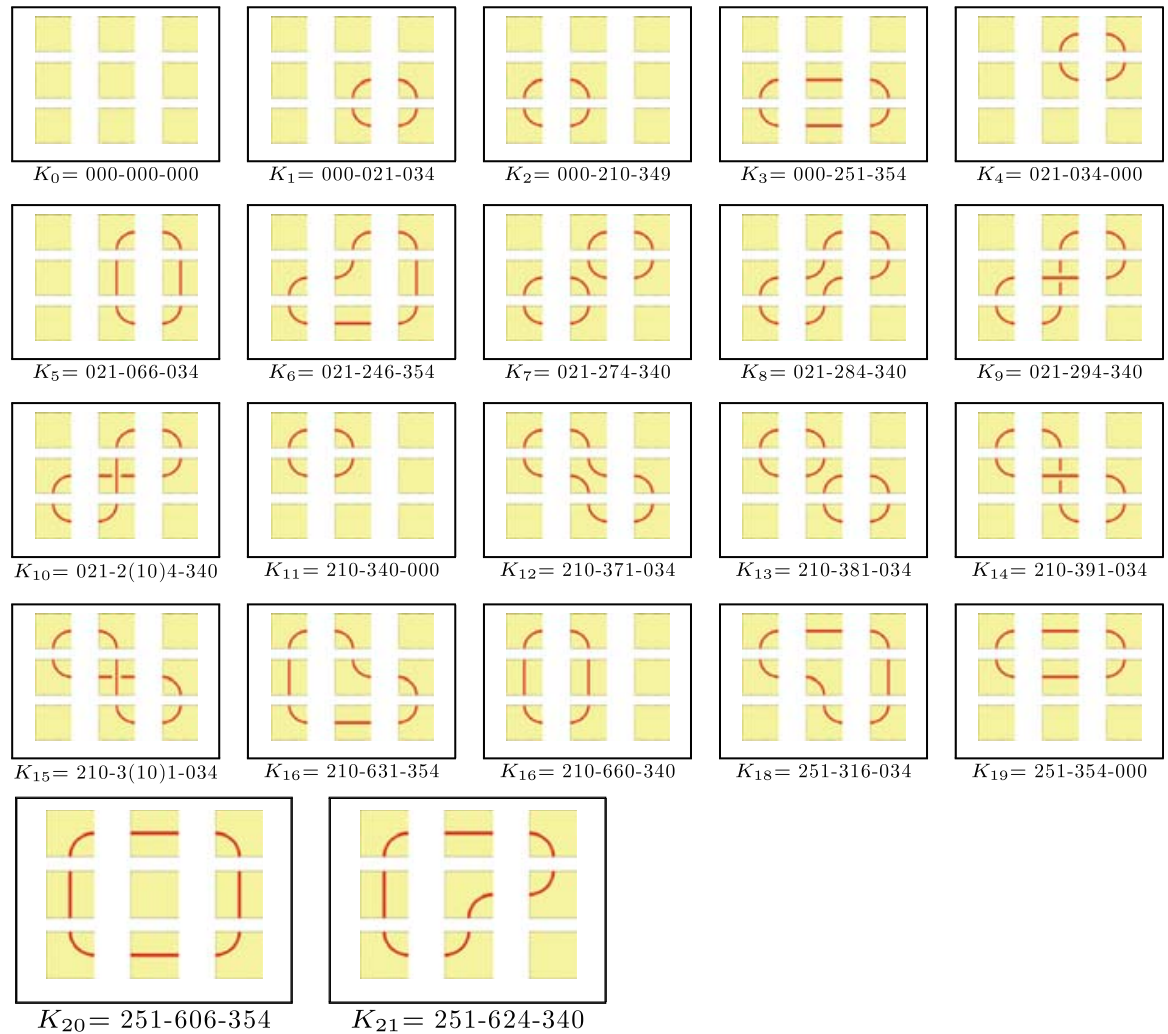

\section{Appendix B: Oriented mosaics and oriented quantum knots}

So far, we have only discussed unoriented objects in this paper, e.g., unoriented mosaics, unoriented knot mosaics, unoriented quantum knots, and so forth. In this appendix, we briefly discuss how all these unoriented objects can be transformed into oriented ones.

Let $\mathbb{T}^{(o)}$ denote the set of the following 29 symbols
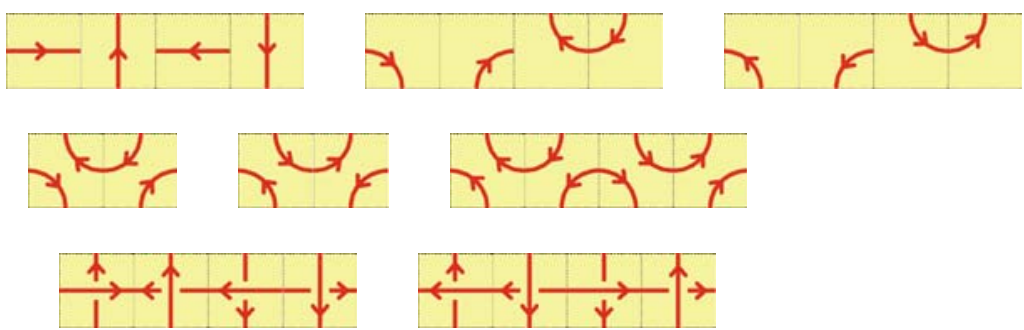
called (oriented) tiles. We often will also denote these tiles respectively by the symbols

$$
T_{0}^{(o)}, T_{1}^{(o)}, T_{2}^{(o)}, \ldots, T_{28}^{(o)}
$$

Moreover, we will frequently omit the superscript ' $(o)$ ' (standing for 'oriented') when it can be understood from context.

Remark 7 Please note that up to rotation there are exactly 9 oriented tiles. The above oriented tiles are grouped according to rotational equivalence.

Definition 11 Let $n$ be a positive integer. We define an (oriented) $n$-mosaic as an $n \times n$ matrix $M=\left(M_{i j}\right)=\left(T_{k(i, j)}\right)$ of (oriented) tiles with rows and columns indexed from 0 to $n-1$. We let $\mathbb{M}^{(n)}$ also denote the set of oriented $n$-mosaics.

Two examples of oriented 4-mosaics are shown below:
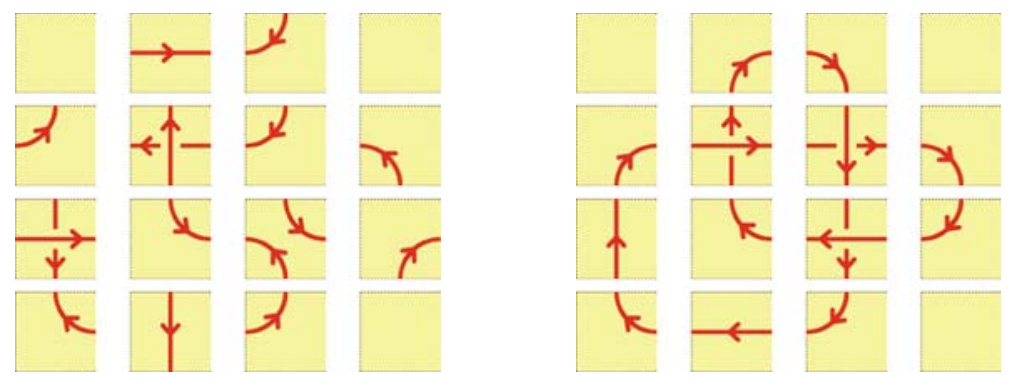

A connection point of an oriented tile is defined as the midpoint of a tile edge which is either the beginning or ending point of an oriented curve drawn on the tile. We define the sign of a connection point as minus (-) or plus (+) accordingly as it is either the beginning point or the ending point of oriented tile curve.

We say that two tiles in an oriented mosaic are contiguous if they lie immediately next to each other in either the same row or the same column. An oriented tile within a oriented mosaic is said to be suitably connected if each its connection points touches a connection point of opposite sign of a contiguous tile.

We are now in a postiion to define what is meant by an oriented mosaic knot:

Definition 12 An oriented knot n-mosaic is an oriented $n$-mosaic in which all tile connection points are suitably connected. We also let $\mathbb{K}^{(n)}$ denote the subset of $\mathbb{M}^{(n)}$ of all oriented $n$-mosaic knots.

The remaining definitions are straight forward, and left to the reader.

\section{References}

1. Aharonov, D., Jones, V., Landau, Z.: On the quantum algorithm for approximating the jones polynomial. http://arxiv.org/abs/quant-ph/0511096

2. Chen, G., Kauffman, L., Lomonaco, S.J. (eds.): The Mathematics of Quantum Computation and Quantum Topology. Chapman \& Hall/CRC (2007)

3. Collins, G.P.: Computing with quantum knots. Sci. Am. April, 56-63 (2006) 
4. Crowell, R.H., Fox, R.H.: Introduction to Knot Theory. Springer-Verlag (1977)

5. Helstrom, C.W.: Quantum Detection and Estimation Theory. Academic Press (1976)

6. Jacak, L., Sitko, P., Wieczorek, K., Wójs, A.: Quantum Hall Systems: Braid groups, Composite Fermions, and Fractional Charge. Oxford University Press (2003)

7. Kauffman, L.H., Lomonaco, S.J.: Quantum knots. Proc. SPIE (2004). http://arxiv.org/abs/quant-ph/ 0403228

8. Kauffman, L.H., Lomonaco, S.J., Jr.: $q$-Deformed spin networks, knot polynomials, and anyonic topological quantum computation. J. Knot Theory 16(3), 267332 (2007). http://xxx.lanl.gov/abs/ quant-ph/0606114

9. Kauffman, L.H., Lomonaco, S.J., Jr.: Spin networks and anyonic topological computing. Proc. SPIE 6244 (2006). http://xxx.lanl.gov/abs/quant-ph/0603131

10. Kauffman, L.H.: Knots and Physics, 3rd edn. World Scientific (2001)

11. Kitaev, A.Y.: Fault-tolerant quantum computation, by anyons. http://arxiv.org/abs/quant-ph/9707021

12. Leonhardt, U.: Measuring the Quantum State of Light. Cambridge University Press (1997)

13. Lickorish, W.B.R.: An Introduction to Knot Theory. Springer (1997)

14. Lomonaco, S.J.: The modern legacies of Thomson's atomic vortex theory in classical electrodynamics. AMS PSAPM/51, Providence, RI, pp. 145-166 (1996)

15. Lomonaco, S.J., Jr., Kauffman, L.H.: Topological quantum computing and the Jones polynomial. Proc. SPIE 6244 (2006). http://xxx.lanl.gov/abs/quant-ph/0605004

16. Lomonaco, S.J., Jr.: A Rosetta stone for quantum mechanics with an introduction to quantum computation. PSAPM, 58, AMS, Providence, RI, pp. 3-65 (2002)

17. Lomonaco, S.J., Jr. (ed.): Quantum computation. PSAPM, 58, American Mathematical Society, Providence, Rhode Island (2002)

18. Lomonaco, S.J., Jr.: Five dimensional knot theory AMS CONM 20, 249-270 (1984). http://www.cs. umbc.edu/ lomonaco/5knots/5knots.pdf

19. Lomonaco, S.J., Jr.: The homotopy groups of knots I. How to compute the algebraic 3-type. Pacific J. Math. 95(2), 349-390 (1981)

20. Murasugi, K.: Knot Theory and Its Applications. Birkhauser (1996)

21. Nielsen, M.A., Chuang, I.L.: Quantum Computation and Quantum Information. Cambridge University Press (2000)

22. Peres, A.: Quantum Theory: Concepts and Methods. Kluwer (1993)

23. Rasetti, M., Regge, T.: Vortices in He II, current algebras and quantum knots. Physica 80 A, NorthHolland, pp. 217-233 (1975)

24. Sakuri J.J.: Modern Quantum Mechanics, revised edn. Addison-Wesley (1994)

25. Shankar, R.: Principles of Quantum Mechanics, 2nd edn. Plenum (1994)

26. Sarma, S.D., Freedman, M., Nayak, C.: Topologically protected qubits from a possible non-Abelian fractional quantum hall state. Phys. Rev. Lett. 94, 166802-1-168802-4 (2005)

27. Shor, P.W., Jordan, S.P.: Estimating Jones polynomials is a complete problem for one clean qubit. http://arxiv.org/abs/0707.2831

28. Wen, X.-G.: Quantum Field Theory of Many-Body Systems. Oxford Press (2004)

29. Zee, A.: Quantum Field Theory in a Nutshell. Princeton University Press (2003)

30. Madison, K.W., Chevy, F., Wohlleben, W., Dalibard, J.: Vortex formation in a stirred Bose-Einstein condensate. Phys. Rev. Lett. 84, 806-809 (2000) 